\title{
Spectra of Wess-Zumino-Witten Models with Arbitrary Simple Groups ${ }^{\star}$
}

\author{
G. Felder ${ }^{1}$, K. Gawẹdzki ${ }^{2}$ and A. Kupiainen ${ }^{3}$ \\ 1 Theoretische Physik, ETH-Hönggerberg, CH-8093 Zürich, Switzerland \\ 2 C.N.R.S., I.H.E.S., F-91440 Bures-sur-Yvette, France \\ 3 Research Institute for Theoretical Physics, University of Helsinki, SF-00170 Helsinki 17, Finland
}

\begin{abstract}
We consider the Wess-Zumino-Witten two-dimensional sigma models with fields taking values in an arbitrary connected (but not necessarily simply connected) simple Lie group $G$. The quantum states of the theory are realized geometrically as sections of a line bundle over the loop group $L G$. The action of the current algebra of the theory is decomposed into highest weight representations by explicit construction of the highest weight states. This solves for the spectrum of the models. As a by-product, we obtain modular invariant partition functions of the theory on tori. The present paper extends the results of [7] where the cases $G=S U(2)$ and $S O(3)$ were treated.
\end{abstract}

\section{Introduction}

The Wess-Zumino-Witten (WZW) models [27,15] of two-dimensional quantum fields with values in group manifolds have attracted considerable attention in recent years. There are multiple reasons for that:

1. In special cases, the WZW models provide a non-abelian extension of bosonization rules [27] whose abelian version had been one of the main tools in the analysis of two-dimensional field theories.

2. Because of extremely rich symmetry combining non-abelian current algebra and conformal invariance, they provide generally non-trivial examples of field theories solvable not only for spectrum but for Green functions as well [15].

3. As models of conformal field theory, the WZW systems are possible building blocks of perturbative string theory vacua [11].

4. Models with rank two groups play an important role in the classification of the so-called minimal conformal field theories $[4,6,10]$.

\footnotetext{
* Ce rapport a été publié en partie grâce à une subvention du Fonds FCAR pour l'aide et le soutien à la rercherche
} 
Last but not least,

5. The WZW models provide a natural meeting- and play-ground for many ideas of modern mathematics and physics like anomalies, Virasoro and Kac-Moody algebras, Yang-Baxter equations, braid groups [8], etc.

The distinctive feature of the WZW theories is that they are defined through an action functional containing a topological term. Our aim in the present paper is the canonical quantization of the models. As pointed out by many authors $[24,28]$, due to the topological character of the theory, the wave functions, instead of being functionals on the configuration space $L G$ of the field ( $L G$ is the space of loops in group $G$ ), become sections of a complex line bundle over $L G$. It is in fact convenient to work in a complexified setup and consider as states the analytic sections of a holomorphic line bundle $L$ over $L G^{\mathrm{C}}$. The classical symmetries of the theory

$$
g \rightarrow g_{1} g g_{2}^{*},
$$

where $g_{i}$ are analytic maps into $G^{\mathrm{C}}$, give rise to a pair of (left, right) projective representations of the loop group $L G^{\mathrm{C}}$ in the space of states or, equivalently, to a pair of representations of the central extension $\widehat{L G}^{\mathbf{C}}$ of $L G^{\mathbf{C}}$, i.e. of the Kac-Moody group [23]. Our main aim in this paper, is to decompose these representations into irreducible highest weight (HW) components. As is well known, this is enough to recover the spectrum of the theory whose Hamiltonian has the Sugawara form $[15,21]$, i.e. is the quadratic expression in the infinitesimal generators of $\widehat{L G}^{\mathrm{C}}$. Using the realization of the quantum states as sections of the line bundle over $L G^{\mathbf{C}}$, we shall be able to construct explicitly the HW wave functionals. First, we do it for the simply connected groups by noting that the HW condition determines the state up to a factor on a dense open subset of the loop group. The weights of both left and right representations of $\widehat{L G}^{\mathrm{C}}$ have to be equal. Only the wave functionals corresponding to the so-called integrable weights extend smoothly to whole $L G^{\mathrm{C}}$. The resulting spectrum of the HW representations has been conjectured in [29] for $G=S U(2)$ and in [11] for a general simple group and in fact it follows from a theorem in [14]. In the next step, we use the result for the simply connected groups to find the HW states for non-simply-connected ones. In the latter case the loop group splits according to the homotopy class of the loops and the space of states splits into different sectors. For each sector, we establish a relation, preserving the HW vectors, between the wave functions in the sector and the original states of the model with the simply connected group. This allows us to find the spectrum in each sector.

For the case of groups $D_{2 p} / \mathbf{Z}_{2} \times \mathbf{Z}_{2}$, there exist two possible quantizations of the WZW model. They correspond to two "periodic vacua" of the theory $[5,12]$ and lead to different contents of the HW representations.

From the calculated spectra of the $\widehat{L G}^{\mathbf{C}}$ representations, we may obtain immediately the partition functions of the models on the tori. We check explicitly their modular invariance employing the techniques developed in [2]. Our list of modular invariants extends the one obtained there. It also agrees with the results of [1] based on a semiclassical analysis in a few cases where the latter ones were 
worked out explicitly. It is worth stressing that we do not use the modular invariance to solve for the spectrum of the theory as advocated in [11]. Instead, we obtain it as a by-product in the theory quantized canonically. Verification of the modular invariance just establishes the absence of global (gravitational) anomalies in the WZW models.

The paper is organized as follows. In Sect. 2, we recall the definition of the model and summarize the main properties of its probability amplitudes with the main stress on their behavior under point-wise multiplication of the fields. In Sect. 3 , we briefly analyze the spectrum of the models with simply connected groups. In Sect. 4, we extend this analysis to non-simply connected groups. In Sect. 5, we prove the modular invariance of the partition functions of the models defined on tori. Finally in Sect. 6, we give explicit formulae for the spectra in all cases of simple non-simply connected groups. Appendix 1 analyzes the quantization conditions for the coupling constant of the models, Appendix 2 establishes the properties of the probability amplitudes of the models stated in Sect. 2 and Appendix 3 collects some useful facts from the Kac-Moody theory.

\section{Probability Amplitudes in the WZW Models}

The WZW theories are usually defined through the action functional [27],

$$
S_{\Sigma}(g)=-\frac{i k}{4 \pi} \int_{\Sigma}\left\langle g^{-1} \partial g, g^{-1} \bar{\partial} g\right\rangle-\frac{i k}{24 \pi} \int_{B}\left\langle\tilde{g}^{-1} d \tilde{g},\left[\tilde{g}^{-1} d \tilde{g}, \tilde{g}^{-1} d \tilde{g}\right]\right\rangle .
$$

The first term in (1) is the standard sigma model action of the field $g: \Sigma \rightarrow G$ (or $G^{\mathrm{C}}$ ), where $\Sigma$ is a compact Riemann surface without boundary (we consider the euclidean version of the model) and $G$ is a compact Lie group, assumed here to be connected and simple but not necessarily simply connected. The second contribution in (1) is the Wess-Zumino topological term: $B$ is a 3-dimensional chain with boundary parametrizing $\Sigma$ and $\tilde{g}$ extends $g$ to $B .\langle\cdot, \cdot\rangle$ denotes the Killing form on the Lie algebra $g$ of $G$ (or on $g^{\mathrm{C}}$ ) normalized so that the longest roots of the algebra have lengths squared two.

Remark 1. The consistency of the quantum theory requires that $S_{\Sigma}(g)$ be well defined (independently of the choice of $\tilde{g}$ ) modulo $2 \pi i \mathbf{Z}$. This implies that

$$
\frac{k}{48 \pi^{2}} \int_{B}\left\langle\tilde{g}^{-1} d \tilde{g},\left[\tilde{g}^{-1} d \tilde{g}, \tilde{g}^{-1} d \tilde{g}\right]\right\rangle \in \mathbf{Z}
$$

for every closed $(\partial B=0) 3$-chain $B$ and imposes a quantization condition on $k: k(>0)$ has to be an integer or an even integer, see Appendix 1.

Remarks 2. There are cases when no extension $\tilde{g}$ may be found. Then a more general definition of the action (see Appendix 2 and [9]) may be used accounting for different consistent choices of $S_{\Sigma}(g)$ for non-extendable $g$ and resulting in different "periodic vacua" of the quantum theory labelled by the elements of the cohomology group $H^{2}(G, U(1))$ [9]. As already mentioned, we shall encounter this phenomenon when studying groups $D_{2 p} / \mathbf{Z}_{2} \times \mathbf{Z}_{2}$. In this case $H^{2}(G, U(1))=\mathbf{Z}_{2}$ and two possible quantizations arise. 
The knowledge of the action of fields defined on surfaces without boundary is enough to set up the vacuum to vacuum transition amplitudes defined (formally) as

$$
\int F(g) e^{-S_{\Sigma}(g)}[d g],
$$

where $[d g]$ denotes the Haar measure on the maps from $\Sigma$ to $G$ and $F(g)$ carries possible insertions.

In order to describe transition amplitudes between general initial and final states, we shall need amplitudes $\exp \left[-S_{\Sigma}(g)\right]$ for Riemann surfaces $\Sigma$ with boundaries. These may be still naturally defined but no more as complex numbers, but as more geometric objects [9]. In the simplest case when the boundary of $\Sigma$ is composed of a single circle, $\partial \Sigma=S^{1}$, the amplitudes $\exp \left[-S_{\Sigma}(g)\right]$ form a complex line bundle $\mathscr{L}$ over $L_{0} G^{\mathrm{C}}$, the set of contractible loops. The base element of $\exp \left[-S_{\Sigma}(g)\right]$ is $g \mid \partial \Sigma$. This might seem strange but what it is saying is quite simple. We may describe $\exp \left[-S_{\Sigma}(g)\right]$ by extending $g$ from the surface $\Sigma$ with boundary to a closed surface and computing the amplitude of the extended map. Different ways of extending $g$ would yield different values related by computable transition functions and there is no canonical way of assigning a complex number to $\exp \left[-S_{\Sigma}(g)\right]$.

The line bundle $\mathscr{L}$ may be extended over the whole $L G^{\mathrm{C}}$. For a general compact Riemann surface $\Sigma$ with the boundary consisting of circles $\gamma_{i}$ and for $g$ mapping $\Sigma$ into $G^{\mathbf{C}}$, the amplitude of $g$

$$
\exp \left[-S_{\Sigma}(g)\right] \in \bigotimes_{i} \mathscr{L}_{g \mid \gamma_{i}}
$$

(is an element of the tensor product of the fibers of $\mathscr{L}$ over the boundary loops of $g$ ).

The essential property of the amplitudes $\exp \left[-S_{\Sigma}(g)\right]$ is their behavior under the multiplication of fields. In the simplest cases, it is described by the Polyakov-Wiegmann (PW) formula [22]

$$
\exp \left[-S_{\Sigma}\left(g_{1} g_{2}\right)\right]=\exp \left[\Gamma_{\Sigma}\left(g_{1}, g_{2}\right)-S_{\Sigma}\left(g_{1}\right)-S_{\Sigma}\left(g_{2}\right)\right]
$$

where

$$
\Gamma_{\Sigma}\left(g_{1}, g_{2}\right)=\frac{i k}{2 \pi} \int_{\Sigma}\left\langle g_{1}^{-1} \bar{\partial} g_{1}, g_{2} \partial g_{2}^{-1}\right\rangle .
$$

Relation (5) holds for $\Sigma=P C^{1}$ or $\Sigma=D \equiv\{z|| z \mid \leqq 1\}$; but in the latter case only if $g_{1}$ or $g_{2}$ is equal 1 on $\partial D$. We shall however need a generalization of (5) to the case $\Sigma=A \equiv\{z|1 \leqq| z \mid \leqq 2\}$, where the boundary contributions have to be taken into account.

For simple, connected, compact groups $G, G=\tilde{G} / Z$, where $\tilde{G}$ is a simply connected group, one of the Cartan series $A_{n}=S U(n+1), B_{n} \cong S O(2 n+1)$, $C_{n} \cong S p(2 n), D_{n} \cong S O(2 n), E_{6}, E_{7}, E_{8}, F_{4}, G_{2}$, and where $Z$ is a subgroup of the center of $\widetilde{G}$. $Z$ may be only trivial for the last three groups and in general is a cyclic group except when it is the center of $D_{2 p}$ equal to $\mathbf{Z}_{2} \times \mathbf{Z}_{2}$. In general, we may write

$$
Z=\left\{e^{2 \pi i m \theta}\right\},
$$

where $m=0,1, \ldots, N-1$ and $\theta$ is an element in the Lie algebra $g$ of $G$ (for $Z=\mathbf{Z}_{N}$ ) 
or $m=\left(m_{1}, m_{2}\right), m_{i}=0$ or $1, m \theta \equiv m_{1} \theta_{1}+m_{2} \theta_{2}, \theta_{i} \in g$ (for $Z=\mathbf{Z}_{2} \times \mathbf{Z}_{2}$ ). Consider maps $h$ and $g$ of the annulus $A$ into $G^{C}$ such that $h \mid \partial A=1$ and

$$
g\left(2 e^{i \varphi}\right)=e^{i \varphi m \theta} \equiv e_{m}(\varphi)
$$

$h$ may be lifted to a unique map $\tilde{h}: A \rightarrow \tilde{G}^{\mathrm{C}}$ such that

$$
\left.\tilde{h}\right|_{|z|=1}=1,\left.\quad \tilde{h}\right|_{|z|=2}=e^{2 \pi i n \theta} \in Z .
$$

The following generalized PW relations hold:

$$
\begin{aligned}
& \exp \left[-S_{A}(h g)\right]=( \pm)^{m \wedge n} \exp \left[-\pi i k\langle m \theta, n \theta\rangle+\Gamma_{A}(h, g)-S_{C P^{1}}(h)-S_{A}(g)\right], \\
& \exp \left[-S_{A}(g h)\right]=( \pm)^{m \wedge n} \exp \left[\pi i k\langle m \theta, n \theta\rangle+\Gamma_{A}(g, h)-S_{C P^{1}}(h)-S_{A}(g)\right],
\end{aligned}
$$

where $m \wedge n \equiv 0$ for $Z=\mathbf{Z}_{N}, m \wedge n \equiv m_{1} n_{2}-m_{2} n_{1}$ for $Z=\mathbf{Z}_{2} \times \mathbf{Z}_{2}$ and $h$ is extended to $C P^{1}$ by setting $h(z)=1$ for $|z| \leqq 1$ or $|z| \geqq 2$. Different choices of sign for $Z=\mathbf{Z}_{2} \times \mathbf{Z}_{2}$ correspond to different periodic vacua.

In Appendix 2, we shall derive relations (10) and (11). The reader not interested in geometric intricacies should notice that (10) and (11) may be used to reconstruct $\mathscr{L}$. Over the component $L_{m} G^{\mathrm{C}}$ of loops homotopic to $e_{m}$, the line bundle $\mathscr{L}$ may be identified [20] with

$$
\left[\operatorname{Map}_{0}\left(A, G^{\mathbf{C}}\right) \times \mathbf{C}^{1}\right] / \operatorname{Map}_{1}\left(A, G^{\mathbf{C}}\right),
$$

where $\operatorname{Map}_{0}\left(A, G^{\mathbf{C}}\right)$ is the set of maps $g: A \rightarrow G^{\mathbf{C}}$ satisfying (8) and $\operatorname{Map}_{1}\left(A, G^{\mathbf{C}}\right)$ contains $h: A \rightarrow G^{\mathrm{C}},\left.h\right|_{\partial A}=1$, which act on $\operatorname{Map}_{0}\left(A, G^{\mathrm{C}}\right)$ by right multiplication and on $\mathbf{C}^{1}$ by multiplication by

$$
( \pm)^{m \wedge n} \exp \left[\pi i k\langle m \theta, n \theta\rangle+\Gamma_{A}(g, h)-S_{C P^{1}}(h)\right] .
$$

The projection onto $L_{m} G^{\mathrm{C}}$ sends $[(g, z)]$ to $g \mid \partial D$.

Remark 1. Notice that the consistency of (10) and (11) requires that for $Z=\mathbf{Z}_{N}$,

$$
\frac{k N}{2}|\theta|^{2} \in \mathbf{Z},
$$

as $n$ is defined modulo $N$. We shall show in Appendix 1 that (13) follows from the quantization condition (2).

Remark 2. Similarly, for $Z=\mathbf{Z}_{2} \times \mathbf{Z}_{2}$ the consistency imposes:

$$
k\left|\theta_{1}\right|^{2}, k\left|\theta_{2}\right|^{2}, k\left\langle\theta_{1}, \theta_{2}\right\rangle \in \mathbf{Z} \text {. }
$$

Here the first two conditions follow from (2). The third one is imposed by demanding that the antiholomorphic involution of $L G^{\mathbf{C}}$ defined by $g \rightarrow g^{*}$ (* $^{*}$ is the complex conjugation on the Lie algebra level) lifts to an antilinear involution of $\mathscr{L}$ (also denoted by *) such that

$$
\exp \left[-S_{\Sigma}\left(g^{*}\right)\right]=\exp \left[-S_{\Sigma}(g)\right]^{*}
$$

see Appendix 2. Condition (15) accounts for the desired positivity properties of the quantum theory.

Given (4), it should be clear how to build general ("stringy") transition 
amplitudes in the WZW theory. The wave functions of the system should be taken as sections $\psi_{i}$ of the line bundle $\mathscr{L}$ over the configuration space $L G$ and the transition amplitudes between states $\psi_{i}$ will be (formally) given by the functional integral (compare (3))

$$
\int \bigotimes_{i} \psi_{i}\left(g \mid \gamma_{i}^{-1}\right) F(g) e^{-S_{\Sigma}(g)}[d g]
$$

where $\Sigma$ is a compact Riemann surface with the boundary composed of circles $\gamma_{i}$. $\gamma_{i}^{-1}$ denotes the circles with inverted orientation. Since the fibers of $\mathscr{L}$ over loops with inverted orientation are naturally dual, the integrand of (20) is a number although individual factors lie in the bundle.

The main subject of the present paper is the analysis of the space of states $\psi_{i}$. For convenience, we shall adopt a slightly more restrictive definition of wave functions admitting only the holomorphic sections of $\mathscr{L}$ over $L G^{\mathrm{C}}$ :

$$
\psi \in \Gamma_{a n}(\mathscr{L}) .
$$

That the quantum states should be defined as sections of a line bundle over the configuration space is a phenomenon known for many mechanical systems and exploited over years by the so-called "geometric quantization" $[17,26]$. Its rules allow one to represent geometrically classical symmetries of the system in the space of quantum states. The projective action of classical symmetry groups on wave functions gives rise to quantum representations of the central extensions of these groups. In our case, the classical symmetries (1.1) act on the configuration space $L G^{\mathrm{C}}$ through the left and right multiplication by loop group elements. The geometric quantization of this action leads to representations of the Kac-Moody group $\widehat{L G} \widehat{C}^{\mathrm{C}}$ (being the central extension of $L G^{\mathrm{C}}$ ) in $\Gamma_{a n}(\mathscr{L})$. We shall define and study these representations in the next two sections.

\section{Spectrum of the WZW Models with Simply Connected Groups}

Throughout this section, we assume that $G$ is simply connected. In this case the WZW amplitudes are consistently and uniquely defined for $k$ integral, see Appendix 1. The Kac-Moody group of quantum symmetries may be constructed using the line bundle $\mathscr{L}$ over $L G^{\mathrm{C}}$. As a set

$$
\widehat{L G}^{\mathrm{C}}=\mathscr{L} \backslash\{\text { zero section }\} .
$$

The multiplication of elements of $\mathscr{L}$ is defined by

$$
\left(\lambda_{1} e^{-S_{D}\left(g_{1}\right)}\right) \cdot\left(\lambda_{2} e^{-S_{D}\left(g_{2}\right)}\right)=\lambda_{1} \lambda_{2} \exp \left[-\Gamma_{D}\left(g_{1}, g_{2}\right)\right] e^{-S_{D}\left(g_{1} g_{2}\right)} .
$$

The reader will convince himself, using the PW formula (2.5), that (2) defines an associative operation on $\mathscr{L}$ under which $\widehat{L G}$ c becomes a group. Embedding $C^{*} \equiv \mathbf{C} \backslash\{0\}$ into $\widehat{L G^{\mathrm{C}}}$ by $\lambda \rightarrow \lambda \exp \left[-S_{D}(1)\right]$, we obtain the exact sequence of groups

$$
1 \rightarrow \mathbf{C}^{*} \rightarrow \widehat{L G}^{\mathbf{C}} \rightarrow L G^{\mathbf{C}} \rightarrow 1 \text {. }
$$

In other words, $\widehat{L G}^{\mathrm{C}}$ is a central extension of $L G^{\mathrm{C}}$. Notice that our construction works for any (integral) $k$. The $k=1$ extension is called the universal one as the other extensions may be obtained from it via modding out the cyclic subgroups 


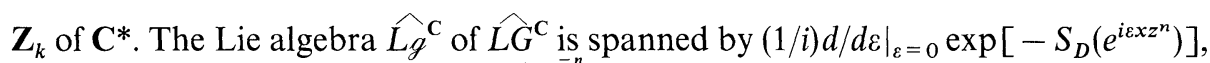
$n \geqq 0$, and $(1 / i) d /\left.d \varepsilon\right|_{\varepsilon=0} \exp \left[-S_{D}\left(e^{i \varepsilon x \bar{z}^{-n}}\right)\right], n \leqq 0$, (both shortly denoted by $x z^{n}$ and identified with the elements of $\left.L^{\mathrm{C}}\right)$ and by $(1 / i) d /\left.d \varepsilon\right|_{\varepsilon=0} e^{i \varepsilon} \exp \left[-S_{D}(1)\right]$ identified with $1 \in \mathbf{C}$. The commutation relations read

$$
\left[x z^{n}, y z^{m}\right]=[x, y] z^{n+m}+n k\langle x, y\rangle \delta_{n+m, 0},
$$

so that $\widehat{\mathrm{Lg}^{\mathrm{C}}}$ is the Kac-Moody algebra with central charge $k[13,21]$.

One can also consider the real form $\widehat{L G}$ of the Kac-Moody group composed of the elements $\hat{g}^{*}=\hat{g}^{-1}$. For $\widehat{L G}$, the exact sequence (3) reduces to

$$
1 \rightarrow U(1) \rightarrow \widehat{L G} \rightarrow L G \rightarrow 1 .
$$

It is easy to represent $\widehat{L G}^{\mathbf{C}} \times \widehat{L G}^{\mathbf{C}}$ in the space of analytic sections $\psi$ on $\mathscr{L}$. For $\hat{g}_{1}, \hat{g}_{2} \in \widehat{L G}^{\mathbf{C}}$ projecting to $g_{1}, g_{2}$ in $L G^{\mathbf{C}}$, we define

$$
\left[l\left(\hat{g}_{1}\right) r\left(\hat{g}_{2}\right) \psi\right](g)=\hat{g}_{1} \cdot \psi\left(g_{1}^{-1} g g_{2}^{*-1}\right) \cdot \hat{g}_{2}^{*} .
$$

It may be shown that representation (6) is the geometric quantization of the action of classical symmetries, see Appendix 2. On the infinitesimal level, it gives two commuting representations of the Kac-Moody algebra generated by $J_{n}(x)=$ $d l\left(x z^{n}\right)$ and $\bar{J}_{n}(x)=-d r\left(x z^{n}\right)$ respectively and the constants. Notice the difference between them: one is complex linear, the other one antilinear although $J_{n}^{a} \equiv J_{n}\left(\tau^{a}\right)$ and $\bar{J}_{n}^{a} \equiv \bar{J}_{n}\left(\tau^{a}\right)$ for $\left(\tau^{a}\right)$ a basis of $g,\left\langle\tau^{a}, \tau^{b}\right\rangle=1 / 2 \delta^{a b},\left[\tau^{a}, \tau^{b}\right]=i f^{a b c} \tau^{c}$, satisfy the same commutation relations:

$$
\left[J_{n}^{a}, J_{m}^{b}\right]=i f^{a b c} J_{n+m}^{c}+\frac{k n}{2} \delta^{a b} \delta_{n+m, 0}
$$

Let us fix some group-theoretic notations [23]. The Cartan subalgebra of $g$ will be denoted by $h$, the Cartan subgroup of $G$ by H. Roots $\alpha$, coroots $\alpha^{v}=2 \alpha /|\alpha|^{2}$ (both in $h$ ) and the step generators $e_{\alpha}=e_{-\alpha}^{*}$ of $G$ satisfy the relations

$$
\begin{aligned}
{\left[h, e_{\alpha}\right] } & =\langle h, \alpha\rangle e_{\alpha}, \quad h \in h, \\
{\left[e_{\alpha}, e_{-\alpha}\right] } & =\alpha^{v} .
\end{aligned}
$$

Positive roots are combinations of simple roots $\alpha_{j}, j=1,2, \ldots, l \equiv \operatorname{rank} G$, with positive coefficients. The Weyl group $W$ of $G$ may be realized as the normalizer $N(H)$ of $H$ in $G$ with $w, w^{\prime} \in N(H), w^{-1} w^{\prime} \in H$, giving the same element of the Weyl group. It is generated by the "reflections in simple roots" $r_{\alpha_{1}}=\exp [(\pi / 2)$ $\left.i\left(e_{\alpha_{j}}+e_{-\alpha_{j}}\right)\right]$. We shall consider nilpotent subgroups $N_{0}^{ \pm}$of $G^{\mathrm{C}}$ generated by $e_{\alpha}, \alpha \gtrless 0$ and the Borel subgroups $B_{0}^{ \pm}=N_{0}^{ \pm} H^{\mathrm{C}}$.

On the loop-group level, it is convenient to extend the Kac-Moody group to the semi-direct product $U(1) \times \widehat{L G}$ associated with the action of $U(1)$ on $\hat{g}=\lambda e^{-S_{D}(g)}$ by rotations of the argument of $g$. On the Lie algebra level, the generator $d$ of $U(1)$ satisfies

$$
\left[d, x z^{n}\right]=n x z^{n}, \quad[d, 1]=0 .
$$

As a vector space, the Lie algebra of $U(1) \times L G$ is $\mathbf{R} d \oplus L g \oplus \mathbf{R}$. Its Cartan subalgebra $\mathbf{R} d \oplus h \oplus \mathbf{R}$ carries the Lorentzian Killing form

$$
\left\langle a d+h+k, a^{\prime} d+h^{\prime}+k^{\prime}\right\rangle=\left\langle h, h^{\prime}\right\rangle+a k^{\prime}+a^{\prime} k .
$$


The affine roots $\tilde{\alpha}=\alpha+n, n$ an integer, and $n, n$ a non-zero integer, correspond to step operators $e_{\tilde{\alpha}}=e_{\alpha} z^{n}$ and $e_{\tilde{\alpha}}=h z^{n}(h$ arbitrary in $h)$, respectively. Positive affine roots correspond to either $n=0, \alpha>0$ or to $n>0$ and $\alpha$ any root or zero. The simple affine roots are $\tilde{\alpha}_{0}=-\phi+1$, where $\phi$ is the highest root of $g$ and $\tilde{\alpha}_{j}=\alpha_{j}$ for $j=1, \ldots, l$. The affine Weyl group $W^{\text {aff }}$ may be realized as a subgroup of $L G$ composed of loops $\varphi \rightarrow e^{2 \pi i \varphi \sigma} w$, where $\sigma$ is in the lattice in $h$ generated by the coroots and $w \in N(H)$ defines an element of the Weyl group $W$. Again $W^{\text {aff }}$ is generated by the "reflections in simple affine roots," $r_{\tilde{\alpha}_{j}}=\exp \left[(\pi / 2) i\left(e_{\tilde{\alpha}_{j}}+e_{-\tilde{\alpha}_{j}}\right)\right]$ (considered as elements of $L G$ ), $r_{\tilde{\alpha}_{0}}(\varphi)=e^{i \varphi \phi^{v}} r_{\phi}$ and $r_{\tilde{\alpha}_{j}}=r_{\alpha_{j}}$ for $j=1, \ldots, l$. We shall consider nilpotent subgroups $N^{ \pm}, M^{ \pm}$of $L G^{\mathbf{C}} . N^{ \pm}$is generated by $e_{\tilde{\alpha}}$ with $\tilde{\alpha} \gtrless 0$, $M^{ \pm}$by $e_{\tilde{\alpha}}$ with $\tilde{\alpha}=\alpha+n$ such that $-\alpha+n \gtrless 0$. They will be identified by means of the lift $g \rightarrow e^{-S_{D}(g)}$ with the subgroups of $L G^{\mathrm{C}}$ composed of the boundary values of holomorphic/antiholomorphic maps $g: D \rightarrow G^{\mathrm{C}}$ with $g(0) \in N_{0}^{ \pm}$(for $N^{ \pm}$) and $g(0) \in N_{0}^{\mp}$ (for $M^{ \pm}$). Similarly, the Borel subgroups $B^{ \pm} \equiv N^{ \pm} H^{\mathrm{C}}$ may be considered as subgroups of $L G^{\mathrm{C}}$ or $\hat{L G}$.

Our aim in the present section is to decompose representation (6) of the pair of Kac-Moody groups into irreducible components generated by the highest weight (HW) vectors $0 \neq \psi \in \Gamma_{a n}(\mathscr{L})$ satisfying, by definition, the relation

$$
\begin{aligned}
J_{n}(x) \psi & =0=\bar{J}_{n}(x) \psi, \quad n>0, \\
J_{0}\left(e_{\alpha}\right) \psi & =0=\bar{J}_{0}\left(e_{-\alpha}\right) \psi, \quad \alpha>0, \\
J_{0}(h) \psi & =\left\langle\omega_{L}, h\right\rangle, \bar{J}_{0}(h) \psi=\left\langle\omega_{R}, h\right\rangle \psi, \quad h \in h,
\end{aligned}
$$

for a pair of weights $\omega_{L}, \omega_{R} \in h$. It will be more convenient to rewrite the above definition in terms of the Kac-Moody group action:

$$
\begin{aligned}
& l\left(g_{1}\right) \psi=\psi=r\left(g_{2}\right) \psi, \quad g_{1} \in N^{+}, \quad g_{2} \in M^{+}, \\
& l\left(e^{i h}\right) \psi=e^{i\left\langle\omega_{L}, h\right\rangle} \psi, \quad r\left(e^{i h}\right) \psi=e^{i\left\langle\omega_{R}, h^{*}\right\rangle} \psi, \quad h \in h^{\mathrm{C}} .
\end{aligned}
$$

We shall show now that these conditions completely fix $\psi$ and also set conditions for possible $\omega_{L}$ and $\omega_{R}$. Our argument parallels the Borel-Weil construction for loop groups, see [23], Sect. 11.3.

Let us first use (13) and (14) to evaluate $\psi$ on special loops, namely those of the form $g_{1} w_{0} g_{2}^{*}$ with $g_{1} \in B^{+}, g_{2} \in M^{+}$and $w_{0} \in N(H)$ representing a Weyl group reflection mapping the positive roots into the negative ones, $w_{0} \alpha w_{0}^{-1}=-\alpha$. The point is that $B^{+} w_{0}\left(M^{+}\right)^{*}=B^{+} w_{0} M^{-}$is an open dense subset of $L G^{\mathrm{C}}$, see below. If $e^{\text {in }}$ is the $H^{\mathrm{C}}$ component of $g_{1}$ then

$$
\psi\left(g_{1} w_{0} g_{2}^{*}\right)=e^{-l\left\langle\omega_{L}, h\right\rangle}\left[l\left(g_{1}\right) r\left(g_{2}\right) \psi\right]\left(g_{1} w_{0} g_{2}^{*}\right)=e^{-l\left\langle\omega_{L}, h\right\rangle} e^{-S_{D}\left(g_{1}\right)} \cdot \psi\left(w_{0}\right) \cdot e^{-S_{D}\left(g_{2}^{*}\right)} .
$$

Setting $\psi\left(w_{0}\right)=$ const $e^{-S_{D}\left(w_{0}\right)}$ and using (2), we obtain

$$
\psi\left(g_{1} w_{0} g_{2}^{*}\right)=\text { const } e^{-\left\langle\left\langle\omega_{L}, h\right\rangle\right.} e^{-S_{D}\left(g_{1} w_{0} g_{2}^{*}\right)} .
$$

Thus the HW condition determines $\psi$ completely (up to a constant factor) on $B^{+} w_{0} M^{-}$and thus everywhere.

On the other hand, since $B^{+} \cap w_{0} M^{-} w_{0}^{-1}=B^{+} \cap N^{-}=1$, given weight $\omega_{L}$, the right-hand side of Eq. (16) defines (up to a multiplicative constant again) an 
analytic section $\psi$ of $\mathscr{L}$ over $B^{+} w_{0} M^{-}$clearly satisfying (13). Moreover, for $h^{\prime} \in h^{\mathrm{C}}$,

$$
\begin{aligned}
{\left[l\left(e^{i h^{\prime}}\right) \psi\right]\left(g_{1} w_{0} g_{2}^{*}\right) } & =e^{-S_{D}\left(e^{i h^{\prime}}\right)} \cdot \psi\left(\left(e^{-i h^{\prime}} g_{1} e^{i h^{\prime}}\right) e^{-i h^{\prime}} w_{0} g_{2}^{*}\right) \\
& =\mathrm{const} e^{-l\left\langle\omega_{L}, h-h^{\prime}\right\rangle} e^{-S_{D}\left(e^{i h^{\prime}}\right)} \cdot e^{-S_{D}\left(e^{-i h^{\prime}} g_{1} w_{0} g_{2}^{*}\right)} \\
& =e^{l\left\langle\omega_{L}, h^{\prime}\right\rangle} \psi\left(g_{1} w_{0} g_{2}^{*}\right),
\end{aligned}
$$

where we have used the fact that $e^{-i h^{\prime}} N^{+} e^{i h^{\prime}}=N^{+}$. Similarly

$$
\begin{aligned}
{\left[r\left(e^{i h^{\prime}}\right) \psi\right]\left(g_{1} w_{0} g_{2}^{*}\right) } & =\psi\left(g_{1} w_{0} e^{i h^{\prime *}}\left(e^{-i h^{\prime}} g_{2} e^{i h^{\prime}}\right) *\right) \cdot e^{-S_{D}\left(e^{-i h^{\prime *}}\right)} \\
& =\psi\left(g_{1} e^{-i h^{\prime *}} w_{0}\left(e^{-i h^{\prime}} g_{2} e^{i h^{\prime}}\right) *\right) \cdot e^{-S_{D}\left(e^{-i h^{\prime *}}\right)} \\
& =e^{-i\left\langle\omega_{L}, h-h^{\prime *}\right\rangle} e^{\left.-S_{D^{(g}} g_{1}{ }^{\prime} g_{2}^{*} e^{i h^{* *}}\right)} \cdot e^{-S_{D}\left(e^{-i h^{\prime *}}\right)} \\
& =e^{l\left\langle\omega_{L} h^{\prime *}\right\rangle} \psi\left(g_{1} w_{0} g_{2}^{*}\right),
\end{aligned}
$$

so that $\psi$ satisfies also (14) with $\omega_{R}=\omega_{L}$.

The above discussion implies that the HW states correspond with multiplicity zero or one to pairs of equal weights $(\omega, \omega)$. The $(\omega, \omega)$ state will appear in the spectrum if and only if $\psi$, as defined by (16) on $B^{+} w_{0} M^{-}$, extends to the analytic section over the whole $L G^{\mathrm{C}}((13)$, and (14) for the extended states will then follow by continuity).

In order to study the limiting behavior of sections $\psi$ given by Eq. (16) on the boundary of $B^{+} w_{0} M^{-}$, we need some facts about the stratification of $L G^{\mathbf{C}}$ (for proofs see [23], Sect. 8.6 and 8.7).

$L G^{\mathrm{C}}$ may be written as the union of disjoint strata

where

$$
L G^{\mathrm{C}}=\bigcup_{\tilde{w} \in W^{\mathrm{aff}}} \Sigma_{\tilde{w}}
$$

$$
\Sigma_{\tilde{w}}=B^{+} \tilde{w} N^{-} \text {. }
$$

The stratification of $L G^{\mathrm{C}}$ which we shall really need is by

$$
\Sigma_{\tilde{w}} w_{0}=B^{+} \tilde{w} w_{0}\left(w_{0}^{-1} N^{-} w_{0}\right)=B^{+} \tilde{w} w_{0} M^{-} .
$$

The reader not familiar with (19) and (20) should consider the case of $G^{\mathrm{C}}=S L(2, \mathbf{C})$ where, by virtue of the Birkhoff theorem [23], any loop can be written as

$$
g_{1}\left(\begin{array}{ll}
z^{n} & 0 \\
0 & z^{-n}
\end{array}\right) g_{2}^{*}, n \in \mathbf{Z},
$$

with $g_{i}$ holomorphic for $|z| \leqq 1$. $W^{\text {aff }}$ may be represented by loops

and

$$
\left(\begin{array}{ll}
z^{n} & 0 \\
0 & z^{-n}
\end{array}\right)
$$

where

$$
\left(\begin{array}{ll}
z^{n} & 0 \\
0 & z^{-n}
\end{array}\right) w_{0},
$$

$$
w_{0}=\left(\begin{array}{ll}
0 & i \\
i & 0
\end{array}\right) \text {. }
$$


Now, stratification (19), (20) may be established in an elementary way with the use of the partition of $S L(2, \mathbf{C})$ into the strata $B_{0}^{+} N_{0}^{-}$and $B_{0}^{+} w_{0} N_{0}^{-}$.

Let us return to the general case. $L G^{\mathrm{C}}$ may be also covered by open dense sets $U_{\tilde{w}}$ indexed by $W^{\text {aff }}$ :

$$
\begin{aligned}
L G^{\mathrm{C}} & =\bigcup_{\tilde{w} \in W^{\text {aff }}} U_{\tilde{w}}, \\
U_{\tilde{w}} & =B^{+} N^{-} \tilde{w} .
\end{aligned}
$$

We have so far found $\psi$ on $\Sigma_{1} w_{0}=U_{1} w_{0}$. Now, we seek to extend it to $\Sigma_{\tilde{w}} w_{0}, \tilde{w} \neq 1$. Some knowledge of the codimensions and relative locations of $\Sigma_{\tilde{w}}$ 's will be needed. In fact, $\Sigma_{\tilde{w}} \subset U_{\tilde{w}}$ and has a finite codimension there. To see this, first note that $N^{-}$ may be split as

$$
N^{-}=\left(N^{-} \cap \tilde{w}^{-1} N^{+} \tilde{w}\right)\left(N^{-} \cap \tilde{w}^{-1} N^{-} \tilde{w}\right) .
$$

On the level of Lie algebras, Eq. (24) is obvious: the first factor is spanned by $e_{\tilde{\beta}}$ with $\widetilde{\beta}=\tilde{w}^{-1}(\tilde{\alpha})<0$ for $\tilde{\alpha}>0$ (by definition $e_{\tilde{w}(\tilde{\alpha})}=\tilde{w} e_{\tilde{\alpha}} \tilde{w}^{-1}$ ) whereas the second factor has $\tilde{\beta}=\tilde{w}^{-1}(\tilde{\alpha})<0$ for $\tilde{\alpha}<0$. The global result holds due to the nilpotency of the groups, see [23], Chapter 8 .

Splitting (24) gives immediately

where

$$
\Sigma_{\tilde{w}}=B^{+} \tilde{w} N_{\tilde{w}}^{-},
$$

$$
N_{\tilde{w}}^{-}=N^{-} \cap \tilde{w}^{-1} N^{-} \tilde{w} .
$$

Moreover, Eq. (25) leads to a unique decomposition of elements of $\Sigma_{w}$.

For $U_{\tilde{w}}$ we need the splitting

where

$$
\tilde{w}^{-1} N^{-} \tilde{w}=N_{\tilde{w}}^{+} N_{\tilde{w}}^{-},
$$

$$
N_{\tilde{w}}^{+}=N^{+} \cap \tilde{w}^{-1} N^{-} \tilde{w} \text {. }
$$

On the level of Lie algebras, Eq. (27) is again clear and the global result follows as before. Splitting (27) yields a unique decomposition

$$
U_{\tilde{w}}=B^{+} \tilde{w} N_{\tilde{w}}^{+} N_{\tilde{w}}^{-} .
$$

Thus $\Sigma_{\tilde{w}} \subset U_{\tilde{w}}$ and its codimension is equal to the dimension of $N_{\tilde{w}}^{+}$. Since $N_{\tilde{w}}^{+}$is generated by $e_{\tilde{\beta}}$ with $\widetilde{\beta}=\tilde{w}^{-1}(\tilde{\alpha})>0$ for $\tilde{\alpha}<0$, its dimension is the number of positive affine roots which $\tilde{w}$ transforms to negative ones, the so-called length $l(\tilde{w})$ of $\tilde{w}$.

We need the following result (see [23], proof of Proposition 11.3.1)

$$
U_{\tilde{w}} \backslash \Sigma_{\tilde{w}} \subset \bigcup_{\substack{\tilde{w}^{\prime} \\ l(\tilde{w})<l(\tilde{w})}} U_{\tilde{w}^{\prime}}
$$

Suppose that our section $\psi$ given by Eq. (16) on $\Sigma_{1} w_{0}=U_{1} w_{0}$ extends analytically to $U_{\tilde{w}} w_{0}$ with $l(\tilde{w})=1$. Then, from Hartogs theorem [16], it follows by induction over $l(\tilde{w})$ that $\psi$ extends uniquely to whole $L G^{\mathrm{C}}$. We are thus left with $l(\tilde{w})=1$.

In this case, $\tilde{w}$ has to be one of the reflections $r_{\tilde{y}_{J}}=\exp \left[(\pi / 2) i\left(e_{\tilde{x}_{j}}+e_{-\tilde{\alpha}_{j}}\right)\right]$ in the simple affine roots $\tilde{\alpha}_{j}$, transforming $\tilde{\alpha}_{j}$ to $-\tilde{\alpha}_{j}$ and keeping the other positive roots positive. Consider thus a general element of $U_{\tilde{w}} w_{0}$ decomposed according 
to (29). As $N_{r_{\tilde{x}_{j}}}^{+}=\left\{\exp \left[-i t e_{\tilde{\alpha}_{j}}\right] \mid t \in \mathbf{C}\right\}$, it may be written as

$$
g_{1} r_{\tilde{\alpha}_{J}} \exp \left[-i t e_{\tilde{\alpha}_{j}}\right] g_{2}^{*} w_{0} \equiv g(t)
$$

where $g_{1} \in B^{+}$and $g_{2}^{*} \in N_{r_{\tilde{\alpha}}}^{-}$. To see that for $t \neq 0, g(t) \in U_{1} w_{0}=B^{+} w_{0} M^{-}$, consider a homomorphism $R_{\tilde{x}_{J}}$ of $S L(2, \mathbf{C})$ into $L G^{\mathrm{C}}$ given by

$$
d R_{\tilde{\alpha}_{j}}\left(\begin{array}{ll}
0 & 1 \\
0 & 0
\end{array}\right)=e_{\tilde{\gamma}_{\jmath}}, \quad d R_{\tilde{\gamma}_{\jmath}}\left(\begin{array}{ll}
0 & 0 \\
1 & 0
\end{array}\right)=e_{-\tilde{\gamma}_{j}}, \quad d R_{\tilde{\gamma}_{\jmath}}\left(\begin{array}{cc}
1 & 0 \\
0 & -1
\end{array}\right)=\alpha_{j}^{v},
$$

where $\alpha_{0}^{v} \equiv-\phi^{v}$ is the projection of $\tilde{\alpha}_{0}^{v}=\left[e_{\tilde{\alpha}}, e_{-\tilde{\gamma}}\right]$ into $L g^{\mathrm{c}}$. For non-zero $t$, we have (compare [23], proof of Proposition 11.3.1)

$$
\begin{aligned}
r_{\tilde{\gamma}_{j}} \exp \left[-i t e_{\tilde{\gamma}_{j}}\right] & =R_{\tilde{\alpha}_{j}}\left(\left(\begin{array}{ll}
0 & i \\
i & 0
\end{array}\right)\left(\begin{array}{cc}
1 & -i t \\
0 & 1
\end{array}\right)\right) \\
& =R_{\tilde{\alpha}_{j}}\left(\left(\begin{array}{ll}
1 & i t^{-1} \\
0 & 1
\end{array}\right)\left(\begin{array}{cc}
t^{-1} & 0 \\
0 & t
\end{array}\right)\left(\begin{array}{ll}
1 & 0 \\
i t^{-1} & 1
\end{array}\right)\right) \\
& =\exp \left[i t^{-1} e_{\tilde{\alpha}_{j}}\right] \exp \left[-\log t \alpha_{j}^{v}\right] \exp \left[i t^{-1} e_{-\tilde{\gamma}_{j}}\right] \\
& \equiv g_{1}(t) e^{i h_{0}(t)} g_{2}(t)^{*}
\end{aligned}
$$

where $g_{1}(t) \in N^{+}, h_{0}(t) \in h^{\mathrm{C}}$ and $g_{2}(t)^{*} \in N^{-}$. Thus for $t \neq 0$,

$$
g(t)=g_{1} g_{1}(t) e^{i h_{0}(t)} g_{2}(t)^{*} g_{2}^{*} w_{0} \in B^{+} w_{0} M^{-},
$$

as $w_{0}^{-1} g_{2}(t)^{*} g_{2}^{*} w_{0} \in M^{-}$. Hence, by virtue of Eq. (16),

$$
\psi(g(t))=\text { const } e^{-\imath\left\langle\omega_{L}, h+h_{0}(t)\right\rangle} \exp \left[-S_{D}(g(t))\right],
$$

where $e^{\text {in }}$ is the $H^{\mathrm{C}}$ component of $g_{1} \in B^{+}$, or

$$
\psi(g(t))=\text { const } e^{-l\left\langle\omega_{L} h+h_{0}(t)\right\rangle} \exp \left[-S_{D}\left(g_{1}\right)\right] \cdot \exp \left[-S_{D}\left(g_{t}\right)\right] \cdot \exp \left[-S_{D}\left(g_{2}^{*} w_{0}\right)\right]
$$

with $g_{t} \equiv g_{1}(t) e^{i h_{0}(t)} g_{2}(t)^{*}$. The behavior of the amplitude $\exp \left[-S_{D}\left(g_{t}\right)\right]$ when $t \rightarrow 0$ may be extracted as follows. By Eq. (A.2.22) of Appendix 2, the covariant $t$-derivative of the amplitude is

$$
\begin{aligned}
\frac{D}{d t} \exp \left[-S_{D}\left(g_{t}\right)\right]= & \left\{\frac{i k}{2 \pi} \int_{D}\left\langle\partial\left(g_{t}^{-1} \frac{\partial g_{t}}{\partial t}\right), g_{t}^{-1} \bar{\partial} g_{t}\right\rangle\right. \\
& \left.-\frac{i k}{4 \pi} \oint_{|z|=1}\left\langle g_{t}^{-1} \frac{\partial g_{t}}{\partial t}, g_{t}^{-1} d g_{t}\right\rangle\right\} \exp \left[-S_{D}\left(g_{t}\right)\right] .
\end{aligned}
$$

Since for $|z|=1$,

$$
g_{t}=R_{\tilde{x}_{j}}\left(\left(\begin{array}{ll}
0 & i \\
i & t
\end{array}\right)\right)
$$

is regular at $t=0$, the only singular behavior of the amplitude comes from the $\int_{D}$ term of (37). Integrating it by parts $\left(\partial\left(g_{t}^{-1} \bar{\partial} g_{t}\right)=0\right)$, we obtain

$$
\frac{D}{d t} \exp \left[-S_{D}\left(g_{t}\right)\right]=\left\{\frac{i k}{2 \pi} \oint_{|z|=1}\left\langle g_{t}^{-1} \frac{\partial g_{t}}{\partial t}, g_{t}^{-1} \bar{\partial} g_{t}\right\rangle+\operatorname{reg}(t)\right\} \exp \left[-S_{D}\left(g_{t}\right)\right]
$$


where reg $(t)$ is analytic at $t=0$. The first term in the bracket vanishes for $j=1, \ldots, l$ as $\bar{\partial} g_{t}=0$ then and is equal to $(k / t)\left\langle e_{\phi}, e_{-\phi}\right\rangle=k / t$ for $j=0$. Thus

$$
\exp \left[-S_{D}\left(g_{t}\right)\right]= \begin{cases}e^{\mathrm{reg}(t)} & j=1, \ldots, l \\ t^{k} e^{\mathrm{reg}(t)}, & j=0\end{cases}
$$

Since $h_{0}(t)=i \log t \alpha_{j}^{v}$, we infer from (36) that $\psi(g(t))$ extends analytically to $t=0$ if and only if

and

$$
\left\langle\omega_{L}, \alpha_{j}^{v}\right\rangle \geqq 0, \quad j=1, \ldots, l
$$

$$
\left\langle\omega_{L}, \phi^{v}\right\rangle \leqq k
$$

The weights satisfying (40) and (41) are called integrable.

We have just proven that the $\mathrm{HW}$ states in $\Gamma_{a n}(\mathscr{L})$ correspond (with multiplicity one) to the pairs of equal integrable weights. This is a result conjectured in [11]. In fact it follows from a theorem of [14]. Note however that our approach provides via Eq. (16) a concrete realization of the $\mathrm{HW}$ vectors $\psi$ :

$$
\psi\left(\left.g_{\mathrm{cl}}\right|_{|z|=1}\right)=\text { const } \chi\left(g_{\mathrm{cl}}(0)\right) \exp \left[-S_{D}\left(g_{\mathrm{cl}}\right)\right]
$$

if $g_{\mathrm{cl}}$ satisfies the classical equation of motion $\partial\left(g_{\mathrm{cl}}^{-1} \bar{\partial} g_{\mathrm{cl}}\right)=0$ on $D$ and $\chi$ is an analytic function on $G^{\mathbf{C}}$, the $\mathrm{HW}$ vector of the left and right regular representations of $G$. This follows from the previous discussion since the classical solutions on $D$ are of the form $g_{1} g_{2}{ }^{*}$, where $g_{i}$ are analytic maps from $D$ to $G^{\mathbf{C}}$. The equality of the left and right highest weights of $\chi$ and condition (40) for them is a part of the classical Peter-Weyl representation theory for compact groups [19]. Condition (41), which cuts out all but a finite number of weights, is imposed, as we have seen, by the regularity of states (42).

Starting from the HW vectors $\psi$, one can generate the "HW modules" carrying the irreducible representations of $\widehat{L G}{ }^{\mathrm{C}} \times \widehat{L G}^{\mathrm{C}}$ by taking the linear combinations of vectors $l\left(\hat{g}_{1}\right) r\left(\hat{g}_{2}\right) \psi$. As follows from the main theorem of [14], the (direct) sum of these HW modules gives a subspace of "strongly regular" states in $\Gamma_{a n}(\mathscr{L})$. By definition, a state is strongly regular if it is invariant with respect to some subgroup of the form $\bigcap \hat{g}_{i} N^{+} \hat{g}_{i}^{-1}$ of $\widehat{L G}{ }^{\mathrm{C}}$ (a finite intersection). We do not know whether the strongly regular states are dense in $\Gamma_{a n}(\mathscr{L})$ in some reasonable topology so that the decomposition into the HW modules decomposes also $\Gamma_{a n}(\mathscr{L})$. It is however easy to see that a possible obstruction: the existence in $\Gamma_{a n}(\mathscr{L})$ of lowest weight states, does not occur.

\section{The Case of Non-Simply Connected Groups}

Let $\widetilde{G}$ be one of the simply connected groups considered above. Let $Q(R)\left(Q\left(R^{v}\right)\right)$ denote the lattice generated by the roots (coroots) of $\tilde{G}$. Both $Q(R)$ and $Q\left(R^{v}\right)$ are subsets of the Cartan algebra $h$ of $\widetilde{G}$. The Cartan subgroup $\widetilde{H}$ of $\widetilde{G}$ may be identified via the exponential map with $h / 2 \pi Q\left(R^{v}\right)$. Let

$$
Q^{*}(R)=\{h \in h \mid\langle h, \alpha\rangle \in \mathbf{Z} \text { for each } \alpha \in Q(R)\} .
$$

The center of $\widetilde{G}$ corresponds to $2 \pi Q^{*}(R) / 2 \pi Q\left(R^{v}\right)\left(h \in 2 \pi Q^{*}(R)\right.$ if and only if $e^{i h}$ 
acts trivially in the adjoint representation). The subgroups $Z$ of the center of $\tilde{G}$ are generated either by a single element $\theta \in Q^{*}(R)$ or by $\theta_{1}, \theta_{2} \in Q^{*}(R)$, see (2.7).

In the present section, we shall consider the WZW models with fields taking values in $G=\widetilde{G} / Z$. The loop group $L G^{\mathbf{C}}$ decomposes into sectors corresponding to different homotopy classes of loops labelled by elements of $Z$. Defining $L_{m} G^{\mathbf{C}}$ as the set of loops homotopic to $e_{m}\left(e_{m}(\varphi) \equiv e^{i \varphi m \theta}\right)$, we have

$$
L G^{\mathrm{C}}=\bigcup_{m} L_{m} G^{\mathrm{C}} \text {. }
$$

Let us denote by $\mathscr{L}_{m}$ the restriction of line bundle $\mathscr{L}$ to $L_{m} G^{\mathrm{C}}$. Recall that for $g$ mapping the annulus $A$ into $G^{\mathrm{C}}, g\left(2 e^{i \varphi}\right)=e_{m}(\varphi)$,

$$
e^{-S_{A}(g)} \in \mathscr{L}_{e_{m}} \otimes \mathscr{L}_{g \mid \partial D}^{-1}
$$

see (2.4). Hence a general element of $\mathscr{L}_{m}$ may be represented as

$$
\mu e^{S_{A}(g)}
$$

where $\mu \in \mathscr{L}_{e_{m}}$. The space of quantum states of the model also decomposes into sectors,

$$
\Gamma_{a n}(\mathscr{L})=\bigoplus_{m} \Gamma_{a n}\left(\mathscr{L}_{m}\right)
$$

The classical symmetries of the theory, acting on the configuration space $L_{m} G^{\mathbf{C}}$ by left and right multiplication by elements of $L \widetilde{G}^{\mathrm{C}}$ lift to left and right actions of the Kac-Moody group $\widetilde{L}_{\widetilde{G}^{\mathrm{C}}}^{\mathrm{c}}$ on $\mathscr{L}_{m}$. These actions may be easily found with the use of rules of the geometric quantization. We shall give here only the final result (see Appendix 2 for simpler infinitesimal expressions):

Upon representing general elements of $\widehat{L}^{\mathbf{C}}$ as $\lambda_{i} \exp \left[-S_{D}\left(g_{i}\right)\right], 0 \neq \lambda_{i} \in \mathbf{C}$, $g_{i}: D \rightarrow \widetilde{G}^{\mathrm{C}}$, their action on an element of $\mathscr{L}_{m}$ given by (4) becomes

$\left(\lambda_{1} \exp \left[-S_{D}\left(g_{1}\right)\right]\right) \cdot\left(\mu \exp \left[S_{A}(g)\right]\right)=\lambda_{1} \exp \left[\Gamma_{A}\left(\tilde{g}_{1}, g\right)-S_{C P^{1}}\left(\tilde{g}_{1}\right)\right] \mu \exp \left[S_{A}\left(\tilde{g}_{1} g\right)\right]$,

$\left(\mu \exp \left[S_{A}(g)\right]\right) \cdot\left(\lambda_{2} \exp \left[-S_{D}\left(g_{2}\right)\right]\right)=\lambda_{2} \exp \left[\Gamma_{A}\left(g, \tilde{g}_{2}\right)-S_{C P^{1}}\left(\tilde{g}_{2}\right)\right] \mu \exp \left[S_{A}\left(g \tilde{g}_{2}\right)\right]$,

where $\tilde{g}_{i}: C P^{1} \rightarrow \widetilde{G}^{\mathrm{C}}$ extend $g_{i}$ so that $\tilde{g}_{i}(z)=1$ for $|z| \geqq 2$ (and should be treated as $G^{\mathrm{C}}$ valued when required by the context). The reader may check, using the PW formulae, that the right-hand sides of (6) and (7) depend only on the line bundle elements on the left-hand sides and that the action has the required associativity properties.

For what follows, it will be important to know how the elements of $Z \subset L \widetilde{G}^{\mathrm{C}}$ act in each sector. Using Eq. (6) and the PW formula (2.10), we obtain for $g_{1}=e^{2 \pi i n \theta}$,

$$
\begin{aligned}
\exp \left[-S_{D}\left(e^{2 \pi i n \theta}\right)\right] \cdot\left(\mu \exp \left[S_{A}(g)\right]\right) & =\exp \left[\Gamma_{A}\left(\tilde{g}_{1}, g\right)-S_{C P^{1}}\left(\tilde{g}_{1}\right)\right] \mu \exp \left[S_{A}\left(\tilde{g}_{1} g\right)\right] \\
& =( \pm)^{m \wedge n} \exp [-\pi i k\langle m \theta, n \theta\rangle] \mu \exp \left[S_{A}(g)\right]
\end{aligned}
$$

so that for $\hat{g} \in \mathscr{L}_{m}$

$$
e^{2 \pi i n \theta} \cdot \hat{g}=( \pm)^{m \wedge n} \exp [-\pi i k\langle m \theta, n \theta\rangle] \hat{g},
$$


and similarly

$$
\hat{g} \cdot e^{2 \pi i n \theta}=( \pm)^{m \wedge n} \exp [\pi i k\langle m \theta, n \theta\rangle] \hat{g} .
$$

The action of $\widehat{L}^{\mathrm{G}} \mathrm{C} \times \widehat{\widetilde{G}}^{\mathrm{c}}$ on $\mathscr{L}_{m}$ induces its representation $l \times r$ on $\psi \in \Gamma_{a n}\left(\mathscr{L}_{m}\right)$ defined again by formula (3.6). Our aim is to decompose $l \times r$ in each sector into HW representations built upon HW states. This may be easily achieved by establishing a correspondence between the states $\psi$ in $\Gamma_{a n}\left(\mathscr{L}_{m}\right)$ and wave functions $\widetilde{\psi}$ of the simply connected problem with group $\widetilde{G}$, i.e. analytic sections of the bundle $\tilde{\mathscr{L}}$ over $L \widetilde{G}^{\mathrm{C}}$. Given $0 \neq \hat{\varepsilon}_{m} \in \mathscr{L}_{m}$ (projecting to $\varepsilon_{m} \in L_{m} G^{\mathrm{C}}$ ), we shall assign to $\psi$ a section $\tilde{\psi}$ satisfying

$$
\hat{\varepsilon}_{m} \cdot \tilde{\psi}(g)=\psi\left(\varepsilon_{m} g\right)
$$

for each $g \in L \tilde{G}^{\mathrm{C}}$. It is easy to see that Eq. (11) defines a unique $\tilde{\psi}$. Moreover, the consistency requires that

$$
\begin{aligned}
\hat{\varepsilon}_{m} \cdot \tilde{\psi}(g)=\hat{\varepsilon}_{m} \cdot \tilde{\psi}\left(e^{-2 \pi i n \theta} g\right) & =\left(\hat{\varepsilon}_{m} \cdot e^{-2 \pi i n \theta}\right) \cdot\left(e^{2 \pi i n \theta} \cdot \tilde{\psi}\left(e^{-2 \pi i n \theta} g\right)\right) \\
& =( \pm)^{m \wedge n} \exp [-\pi i k\langle m \theta, n \theta\rangle] \hat{\varepsilon}_{m} \cdot\left[l\left(e^{2 \pi i n \theta}\right) \tilde{\psi}\right](g),
\end{aligned}
$$

where we have used multiplication rule (10). Hence

$$
l\left(e^{2 \pi i n \theta}\right) \tilde{\psi}=( \pm)^{m \wedge n} \exp [\pi i k\langle m \theta, n \theta\rangle] \tilde{\psi} .
$$

Conversely, since $\varepsilon_{m} L \widetilde{G}^{\mathrm{C}} / Z=L_{m} G^{\mathrm{C}}$, every analytic section of $\tilde{\mathscr{L}}$ satisfying (13) defines via (11) a unique analytic section $\psi$ of $\mathscr{L}_{m}$. $\psi \rightarrow \widetilde{\psi}$,

Let us find out how the Kac-Moody action behaves under the transformation

$$
\begin{aligned}
{\left[l\left(\hat{g}_{1}\right) r\left(\hat{g}_{2}\right) \psi\right]\left(\varepsilon_{m} g\right) } & =\hat{g}_{1} \cdot \psi\left(g_{1}^{-1} \varepsilon_{m} g g_{2}^{*-1}\right) \cdot \hat{g}_{2}^{*} \\
& =\hat{g}_{1} \cdot \hat{\varepsilon}_{m} \cdot \widetilde{\psi}\left(\varepsilon_{m}^{-1} g_{1}^{-1} \varepsilon_{m} g g_{2}^{*-1}\right) \cdot \hat{g}_{2}^{*} .
\end{aligned}
$$

Let $g_{1}^{\prime}=\varepsilon_{m}^{-1} g_{1} \varepsilon_{m}$ and let $\hat{g}_{1}^{\prime} \in \widehat{L} \widetilde{G}^{\mathrm{C}}$ be the unique element projecting to $g_{1}^{\prime} \in L \widetilde{G}^{\mathrm{C}}$ such that

$$
\hat{g}_{1} \cdot \hat{\varepsilon}_{m}=\hat{\varepsilon}_{m} \cdot \hat{g}_{1}^{\prime} .
$$

The right-hand side of Eq. (14) may be written as $\hat{\varepsilon}_{m} \cdot\left[l\left(\hat{g}_{1}^{\prime}\right) r\left(\hat{g}_{2}\right) \tilde{\psi}\right](g)$ resulting in the relation

$$
\left[l\left(\hat{g}_{1}\right) r\left(\hat{g}_{2}\right) \psi\right]^{\sim}=l\left(\hat{g}_{1}^{\prime}\right) r\left(\hat{g}_{2}\right) \tilde{\psi}
$$

Equation (16) implies that the Kac-Moody modules generated by $\psi$ and $\tilde{\psi}$ are mapped into each other under the transformation $\psi \rightarrow \widetilde{\psi}$.

Up to now, $\hat{\varepsilon}_{m}$ was an arbitrary non-zero element in $\mathscr{L}_{m}$. The crucial fact is however that it may be chosen so that the transformation $\psi \rightarrow \tilde{\psi}$ maps HW states onto the states with the same property. We shall look for $\varepsilon_{m}$ of the form

$$
\varepsilon_{m}=e_{m} \tilde{w}_{m}
$$

where $\tilde{w}_{m}$ is an element of the affine Weyl group $W^{\text {aff }}$. More explicitly,

$$
\varepsilon_{m}(\varphi)=e^{i \varphi\left(m \theta+\sigma_{m}\right)} w_{m} \equiv e^{-l \varphi \omega_{\mu_{m}(0)}} w_{m},
$$

where $w_{m} \in \widetilde{G}$ represents an element of the Weyl group of $\widetilde{G}$ and $\sigma_{m}$ is in the coroot lattice $Q\left(R^{v}\right) \subset h$. The complicated notation for $m \theta+\sigma_{m}$ will become transparent later. Notice that, since $\varphi \rightarrow e^{i \varphi \sigma_{m}}$ lifts to a loop in $\widetilde{G}, \varepsilon_{m}$ is homotopic to $e_{m}$ and 
thus lies in $L_{m} G$. In order to be able to compare the HW conditions (3.13), (3.14) for $\psi$ and $\tilde{\psi}$, we would like to assure that

and

$$
\varepsilon_{m}^{-1} \tilde{H}^{\mathrm{C}} \varepsilon_{m}=\tilde{H}^{\mathrm{C}}
$$

$$
\varepsilon_{m}^{-1} \tilde{N}^{+} \varepsilon_{m}=\tilde{N}^{+} .
$$

Relation (19) holds automatically since $w_{m}$ normalizes $\widetilde{H}^{\mathrm{C}}$.

Let us discuss condition (20). The Lie algebra of the nilpotent group $\tilde{N}^{+}$is generated by the step generators $e_{\tilde{\alpha}}$ of the Kac-Moody algebra with $\tilde{\alpha}=\alpha+n>0$. Let us see how they transform under the conjugation by $\varepsilon_{m}^{-1}$ :

$$
\begin{aligned}
\varepsilon_{m}(\varphi)^{-1} e_{\alpha} e^{i n \varphi} \varepsilon_{m}(\varphi) & =w_{m}^{-1} e^{i \varphi \omega_{\mu_{m}(0)}} e_{\alpha} e^{-i \varphi \omega_{\mu_{m}(0)}} w_{m} e^{i n \varphi} \\
& =w_{m}^{-1} e_{\alpha} w_{m} \exp \left[i\left(n+\left\langle\alpha, \omega_{\mu_{m}(0)}\right\rangle\right) \varphi\right] \\
& =e_{w_{m}}^{-1}(\alpha) \exp \left[i\left(n+\left\langle\alpha, \omega_{\mu_{m}(0)}\right\rangle\right) \varphi\right],
\end{aligned}
$$

where $w_{m}^{-1}(\alpha) \equiv w_{m}^{-1} \alpha w_{m}$. Hence

with

$$
\varepsilon_{m}^{-1} e_{\alpha+n} \varepsilon_{m}=e_{\alpha^{\prime}+n^{\prime}}
$$

$$
\alpha^{\prime}=w_{m}^{-1}(\alpha), \quad n^{\prime}=n+\left\langle\alpha, \omega_{\mu_{m}(0)}\right\rangle
$$

(this also holds for $\alpha=0$ ).

In Appendix 3, we prove that it is possible to choose $\tilde{w}_{m} \in W^{\text {aff }}$ so that $\alpha^{\prime}+n^{\prime}$ is positive if and only if so is $\alpha+n$. We also establish there some useful relations involving $\tilde{w}_{m}$. For the corresponding choice of $\varepsilon_{m}$, condition (20) follows.

Let us elucidate somewhat the problem of choice of $\tilde{w}_{m}$ by relating it to some facts in the Kac-Moody theory. By letting the affine roots act on the Cartan algebra by

$$
(\alpha+n)(h)=\langle\alpha, h\rangle+n,
$$

we may characterize the positive affine roots as those positive on the positive Weyl alcove

Since

$$
C_{0}^{\text {aff }}=\{h \in h \mid \tilde{\alpha}(h)>0 \text { for } \tilde{\alpha}>0\} .
$$

$$
\left(\alpha^{\prime}+n^{\prime}\right)(h)=\left\langle\alpha, w_{m}(h)+\omega_{\mu_{m}(0)}\right\rangle+n=(\alpha+n)\left(w_{m}(h)+\omega_{\mu_{m}(0)}\right),
$$

$\alpha^{\prime}+n^{\prime}$ are positive for positive $\alpha+n$ if and only if

$$
w_{m}\left(C_{0}^{\text {aff }}\right)+\omega_{\mu_{m}(0)}=C_{0}^{\text {aff }} .
$$

Relation (27) may be rewritten as

$$
\tilde{w}_{m}^{-1}\left(C_{m}^{\text {aff }}\right) \equiv w_{m}^{-1}\left(C_{m}^{\text {aff }}+\sigma_{m}\right)=C_{0}^{\text {aff }},
$$

where $C_{m}^{\text {aff }} \equiv C_{0}^{\text {aff }}+m \theta$ is another Weyl alcove (i.e. a connected component of $h \backslash\{h \mid\langle\alpha, h\rangle \in \mathbf{Z}$ for some root $\alpha\})$.

Now, the existence of $\tilde{w}_{m}$ satisfying (28) (unique up to multiplication of $w_{m}$ by an element of the Cartan subgroup) follows from the simple transitivity of the affine Weyl group on the set of Weyl alcoves [23, Proposition 5.14]. The Weyl alcove $C_{0}^{\text {aff }}$ is a simplex with vertices $0,1 / k_{1}^{v} \omega_{1}, \ldots, 1 / k_{l}^{v} \omega_{l}$, where $\omega_{1}, \ldots, \omega_{l}$ are 
the fundamental weights of the group, $\left\langle\omega_{i}, \alpha_{j}^{v}\right\rangle=\delta_{i j}$, and $k_{i}^{v}$ are the dual Kac labels appearing in the decomposition of the highest coroot $\phi^{v}=\Sigma k_{j}^{v} \alpha_{j}^{v}$. We shall put $\omega_{0}=0$ and $k_{0}^{v}=1$. The transformation $h \rightarrow w_{m}(h)+\omega_{\mu_{m}(0)}$ satisfying (27) has to rearrange the vertices of $C_{0}^{\text {aff }}$. As the ratios of the dual Kac labels of the interchanged vertices are easily seen to be integer, only the vertices with equal dual Kac labels may be exchanged. Thus

$$
w_{m}\left(\omega_{j}\right)+k_{j}^{v} \omega_{\mu_{m}(0)}=\omega_{\mu_{m}(j)}
$$

for some permutation $\left(\mu_{m}(0), \mu_{m}(1), \ldots, \mu_{m}(l)\right)$ preserving the values of $k_{j}^{v}$ 's. Note that (29) is consistent with the previous definition of $\omega_{\mu_{m}(0)}$. This establishes a relation between the elements of the center of group $\widetilde{G}$ and the symmetries of the extended Dynkin diagram of the Lie algebra of $\widetilde{G}$.

In order to relate the HW states $\psi$ and $\tilde{\psi}$, we still need more than relations (19) and (20), see (16). Any $g_{1} \in \widetilde{B}^{+}=\tilde{H}^{\mathrm{C}} \tilde{N}^{+}$is the boundary value of an analytic map from $D$ into $\widetilde{G}^{\mathrm{C}}$ (which we denote by the same symbol). Relations (19) and (20) imply that also $g_{1}^{\prime}=\varepsilon_{m}^{-1} g_{1} \varepsilon_{m}$ extends analytically to $D$. For $\hat{g}_{1}=\exp \left[-S_{D}\left(g_{1}\right)\right]$, we have to compute $\hat{g}_{1}^{\prime}$ as given by Eq. (15). Since $g_{1} \rightarrow \hat{g}_{1}^{\prime}$ is a group homomorphism,

$$
\hat{g}_{1}^{\prime}=\chi\left(g_{1}\right) \exp \left[-S_{D}\left(g_{1}^{\prime}\right)\right]
$$

for some one-dimensional representation $\chi$ of $\tilde{B}^{+}$(both sides project to $g_{1}^{\prime}$ ). Representing $\hat{\varepsilon}_{m}$ as $\mu \exp \left[S_{A}\left(\tilde{\varepsilon}_{m}\right)\right]$, where $\tilde{\varepsilon}_{m}: A \rightarrow G^{\mathrm{C}}$ interpolates between $\varepsilon_{m}$ for $|z|=1$ and $e_{m}$ for $|z|=2$, we obtain using definition (6):

$$
\hat{g}_{1} \cdot \hat{\varepsilon}_{m}=\exp \left[\Gamma_{A}\left(\tilde{g}_{1}, \tilde{\varepsilon}_{m}\right)-S_{C P^{1}}\left(\tilde{g}_{1}\right)\right] \mu \exp \left[S_{A}\left(\tilde{g}_{1} \tilde{\varepsilon}_{m}\right)\right] \text {. }
$$

Similarly,

$$
\hat{\varepsilon}_{m} \cdot \hat{g}_{1}^{\prime}=\chi\left(g_{1}\right) \exp \left[\Gamma_{A}\left(\tilde{\varepsilon}_{m}, \tilde{g}_{1}^{\prime}\right)-S_{C P^{1}}\left(\tilde{g}_{1}^{\prime}\right)\right] \mu \exp \left[S_{A}\left(\tilde{\varepsilon}_{m} \tilde{g}_{1}^{\prime}\right)\right] .
$$

Choosing $\tilde{g}_{1}^{\prime}$ equal to (the analytic extension of) $g_{1}^{\prime}$ on $D$, to $\tilde{\varepsilon}_{m}^{-1} \tilde{g}_{1} \tilde{\varepsilon}_{m}$ on $A$ and to 1 for $|z| \geqq 2$, we find $\chi$ :

$$
\chi\left(g_{1}\right)=\exp \left[\Gamma_{A}\left(\tilde{g}_{1}, \tilde{\varepsilon}_{m}\right)-\Gamma_{A}\left(\tilde{\varepsilon}_{m}, \tilde{g}_{1}^{\prime}\right)-S_{C P^{1}}\left(\tilde{g}_{1}\right)+S_{C P^{1}}\left(\tilde{g}_{1}^{\prime}\right)\right] .
$$

In order to express $\chi$ more explicitly, let us calculate $d / d t \chi\left(e^{i t X}\right)$ at $t=0$ for $X$ in the Lie algebra of $\widetilde{B}^{+}$, i.e. the boundary value of an analytic map from $D$ to $g$ with $X(0)$ in the span of $h^{\mathrm{C}}$ and $e_{\alpha}, \alpha>0$.

Denoting by $\tilde{X}$ an extension of $X$ to $C P^{1}$ vanishing for $|z| \geqq 2$, we obtain

$$
\begin{aligned}
\left.\frac{d}{d t}\right|_{t=0} \chi\left(e^{i t X}\right) & =\left.\frac{d}{d t}\right|_{t=0}\left[\Gamma_{A}\left(e^{i t \tilde{X}}, \tilde{\varepsilon}_{m}\right)-\Gamma_{A}\left(\tilde{\varepsilon}_{m}, \tilde{\varepsilon}_{m}^{-1} e^{i t \tilde{X}} \tilde{\varepsilon}_{m}\right)\right] \\
& =-\frac{k}{2 \pi} \int_{A}\left[\left\langle\bar{\partial} \tilde{X}, \tilde{\varepsilon}_{m} \partial \tilde{\varepsilon}_{m}^{-1}\right\rangle+\left\langle\tilde{\varepsilon}_{m}^{-1} \bar{\partial} \tilde{\varepsilon}_{m}, \partial\left(\tilde{\varepsilon}_{m}^{-1} \tilde{X} \tilde{\varepsilon}_{m}\right)\right\rangle\right] \\
& =-\frac{k}{2 \pi} \int_{A} d\left\langle\tilde{X}, \tilde{\varepsilon}_{m} d \tilde{\varepsilon}_{m}^{-1}\right\rangle=\frac{k}{2 \pi} \oint_{|z|=1}\left\langle\tilde{X}, \varepsilon_{m} d \varepsilon_{m}^{-1}\right\rangle \\
& =\frac{i k}{2 \pi} \oint_{|z|=1}\left\langle\tilde{X}, \omega_{\mu_{m}(0)}\right\rangle \frac{d z}{i z}=i k\left\langle X(0), \omega_{\mu_{m}(0)}\right\rangle,
\end{aligned}
$$


where in the last step we have used the analyticity of $\tilde{X}$ on $D$. Notice that the right-hand side of (34) selects only the $h^{\mathrm{C}}$-component of $X(0)$. In particular, it follows that

and

$$
\chi\left(g_{1}\right)=1 \text { for } \quad g_{1} \in \tilde{N}_{1}^{+}
$$

$$
\chi\left(e^{i h_{1}}\right)=\exp \left[i k\left\langle h_{1}, \omega_{\mu_{m}(0)}\right\rangle\right] \text { for } h_{1} \in h^{\mathbf{C}} .
$$

Given (19), (20), (30) and (36), relation (16) implies that $\psi$ is a HW vector (see $(3.13,14))$ if and only if $\tilde{\psi}$ is. Since, by Sect. 3 , the HW states $\tilde{\psi}$ correspond (with multiplicity 1$)$ to the pairs of equal integrable weights $(\omega, \omega)$ satisfying additionally (see (13))

$$
\langle\omega, n \theta\rangle=k / 2\langle m \theta, n \theta\rangle(+1 / 2 m \wedge n) \bmod [1]
$$

for each $n$, we infer that the HW states $\psi$ are labelled (also with multiplicity 1 ) by pairs of weights $\left(\omega_{L}, \omega_{R}\right)$ where

and

$$
\omega_{L}=w_{m}(\omega)+k \omega_{\mu_{m}(0)} \equiv \mu_{m}(\omega)
$$

$$
\omega_{R}=\omega
$$

This establishes the classification of the HW vectors in each sector of the WZW model with group $G=\widetilde{G} / Z$. The decomposition of $\Gamma_{a n}\left(\mathscr{L}_{m}\right)$ into the HW modules generated by those states follows in-as-much as it was established for the simply connected case, i.e. in the subspace of strongly regular states of [14].

The spectrum of the HW states may be encoded in the "mass matrix" $\left(M_{\omega_{L}, \omega_{R}}\right)$ giving the multiplicities of the $\mathrm{HW}$ vectors corresponding to weights $\left(\omega_{L}, \omega_{R}\right)$. Denote by $C_{m}^{k}$ the set of integrable weights satisfying (37). Our results may be summarized in the formula

$$
M_{\omega_{L}, \omega_{R}}=\sum_{m} \sum_{\omega \in C_{m}^{k}} \delta_{\omega_{L}, \mu_{m}(\omega)} \delta_{\omega, \omega_{R}}
$$

Notice that $\mu_{m}$ defined by (38) carries integrable weights into integrable ones. This follows as the integrable weights are exactly the ones in the closure $k \overline{C_{0}^{\text {aff }}}$ of the positive Weyl alcove dilated by $k$ and the latter is preserved by $\mu_{m}$ due to (27). Without loss of generality, we may then restrict $M_{\omega_{L}, \omega_{R}}$ to integrable weights. Notice also that $\mu_{m}$ maps $C_{m}^{k}$ onto $C_{-m}^{k}$. Indeed,

$$
\left\langle\mu_{m}(\omega), n \theta\right\rangle=\left\langle w_{m}(\omega)+k \omega_{\mu_{m}(0)}, n \theta\right\rangle=\langle\omega, n \theta\rangle-k\langle m \theta, n \theta\rangle \bmod [1]
$$

as $w_{m}(\omega)-\omega$ is in the root lattice and $k \omega_{\mu_{m}(0)}+k m \theta$ is in the coroot lattice, and as such both have integral scalar products with $n \theta$. Now, since $\mu_{m}^{-1}=\mu_{-m}$,

$$
M_{\omega_{L}, \omega_{R}}=\sum_{m} \sum_{\omega \in C_{-m}^{k}} \delta_{\omega_{L}, \omega} \delta_{\mu_{m}^{-1}(\omega), \omega_{R}}=M_{\omega_{R}, \omega_{L}},
$$

i.e. matrix $M$ is symmetric.

The condition $\omega \in C_{m}^{k}$ in (40) may be imposed by writing the characteristic function of $C_{m}^{k}$ as the Fourier sum on the group $Z$ :

$$
1_{C_{m}^{k}}(\omega)=\frac{1}{|Z|} \sum_{n} \exp [-2 \pi i\langle\omega, n \theta\rangle+\pi i k\langle m \theta, n \theta\rangle(+\pi i m \wedge n)] .
$$


Thus

$$
M_{\omega, \omega^{\prime}}=\frac{1}{|Z|} \sum_{m, n} \exp \left[-2 \pi i\left\langle\omega^{\prime}, n \theta\right\rangle+\pi i k\langle m \theta, n \theta\rangle(+\pi i m \wedge n)\right] \delta_{\omega, \mu_{m}\left(\omega^{\prime}\right)} .
$$

We shall compute $M$ more explicitly for all possible cases in Sect. 6 .

The knowledge of the HW content of the space of states for the WZW models gives immediately the spectrum of their Hamiltonian which has the Sugawara form

$$
\mathscr{H}=L_{0}+\bar{L}_{0},
$$

where

$$
L_{0}=\frac{1}{k+h^{v}}\left[\sum_{n=-\infty}^{\infty} \sum_{a}: J_{-n}^{a} J_{n}^{a}:-\frac{1}{24} k \operatorname{dim} G\right],
$$

and $\bar{L}_{0}$ is given by the same formula with $\bar{J}$ 's replacing $J$ 's.

$$
h^{v} \equiv \sum_{j=0}^{l} k_{j}^{v}
$$

is the dual Coxeter number of $G$ and the normal ordering in (46) puts $J_{n}^{a}$ with positive $n$ to the right of the ones with negative $n$. For the HW state $\psi$ characterized by weights $\left(\omega_{L}, \omega_{R}\right)$, we find

$$
\mathscr{H} \psi=\left(k+h^{v}\right)^{-1}\left(\operatorname{Cas}\left(\omega_{L}\right)+\operatorname{Cas}\left(\omega_{R}\right)-\frac{1}{12} k \operatorname{dim} G\right) \psi,
$$

where $\operatorname{Cas}(\omega)$ denotes the value of the quadratic Casimir of $G$ in the HW representation of weight $\omega$. Each operator $J^{a}{ }_{-n}$ or $\bar{J}^{a}{ }_{n}, n \geqq 0$ increases the energy by $n$.

Another important quantity fixed by the HW content of the space of states is the partition function on the torus $T_{\tau}^{2}=\left\{\left(e^{i \varphi_{1}}, e^{i \varphi_{2}}\right)\right\}$ with the complex structure defined by $z=\varphi_{1}+\tau \varphi_{2}, \operatorname{lm} \tau>0$,

$$
Z(\tau)=\operatorname{Trace} e^{2 \pi i \tau L_{0}} e^{-2 \pi i \overline{L_{0}}}
$$

In each $\mathrm{HW}$ representation of weight $\omega$,

$$
\operatorname{Trace}_{\omega} e^{2 \pi i \tau L_{0}}=\operatorname{ch}[k, \omega](\tau),
$$

where the right-hand side is the (restricted) affine character of the representation expressible in terms of Jacobi $\theta$ functions, see $[2,23]$. $Z(\tau)$ may be rewritten in terms of the mass matrix $M$ of the model and the affine characters as

$$
Z(\tau)=\sum_{\omega_{L}, \omega_{R}} \operatorname{ch}\left[k, \omega_{L}\right](\tau) M_{\omega_{L}, \omega_{R}} \overline{\operatorname{ch}\left[k, \omega_{R}\right](\tau)} .
$$

Since $M$ is positive, $Z(\tau)$ is real and positive, as it should.

\section{Modular Invariance}

The partition function $Z(\tau)$ is formally given by the functional integral

$$
\int \exp \left[-S_{T_{\tau}^{2}}(g)\right][d g] \text {. }
$$


The modular automorphisms $A=\left(\begin{array}{ll}a & b \\ c & d\end{array}\right) \in S L(2, \mathbf{Z})$ act on the torus by

$$
\left(\varphi_{1}, \varphi_{2}\right) \stackrel{A}{\rightarrow}\left(a \varphi_{1}+b \varphi_{2}, c \varphi_{1}+d \varphi_{2}\right)
$$

and change its modular ratio by

$$
\tau \rightarrow \tau^{\prime}=\frac{a \tau-b}{-c \tau+d}
$$

The amplitudes are covariant under diffeomorphisms of the Riemann surface, see Appendix 2 or [9], so that

$$
\exp \left[-S_{T_{\tau}^{2}}(g \circ A)\right]=\exp \left[-S_{T_{\tau^{\prime}}^{2}}(g)\right]
$$

Since the Haar measure is invariant under $g \rightarrow g \circ A$, we should expect the modular invariance of the partition function:

$$
Z(\tau)=Z\left(\tau^{\prime}\right)
$$

Indeed, we shall show that (5) holds so that, as expected, no global anomalies appear when the formal integral (1) is computed using the loop-group theory.

We shall base heavily on [2]. It is enough to check that

$$
Z(\tau)=Z(\tau+1)
$$

and

$$
Z(\tau)=Z\left(-\frac{1}{\tau}\right)
$$

Since the modular transformations act linearly in the space of affine characters, conditions (6) and (7) impose requirements on the mass matrices $M$. Here it will be convenient to consider $M$ defined by (4.44) as a matrix labelled by weights in $\left(k+h^{v}\right) \overline{C_{0}^{\text {aff }}}-\rho \supset k \overline{C_{0}^{\text {aff }}}$, where $\rho=\omega_{1}+\cdots+\omega_{l}$. Notice that, due to (4.29),

$$
\rho-w_{m}(\rho)=\rho-\sum_{j=1}^{l} \omega_{\mu_{m}(j)}+\left(\sum_{j=1}^{l} k_{j}^{v}\right) \omega_{\mu_{m}(0)}=h^{v} \omega_{\mu_{m}(0)},
$$

and consequently $\mu_{m}$ maps $\left(k+h^{v}\right) \overline{C_{0}^{\text {aff }}}-\rho$ into itself. The extension of $M$ leaves it symmetric and moreover does not change $Z(\tau)$ of $(4.50)$ since the affine characters vanish for the added weights.

Now, as shown in [2], Eq. (6) holds provided

$$
M_{\omega, \omega^{\prime}}=0 \quad \text { if } \quad|\tilde{\omega}|^{2} \neq\left|\tilde{\omega}^{\prime}\right|^{2} \bmod \left[2\left(k+h^{v}\right)\right] \text {, }
$$

where $\tilde{\omega} \equiv \omega+\rho$. To establish (9), we shall need the following property of $\omega_{\mu_{m}(0)}$ proven in Appendix 3.

$$
2\left\langle\rho, \omega_{\mu_{m}(0)}\right\rangle=h^{v}\left|\omega_{\mu_{m}(0)}\right|^{2} \in \mathbf{Z} .
$$

Now $M_{\omega, \omega^{\prime}} \neq 0$ if and only if for some $m, \omega^{\prime}$ satisfies $(4.37)$ and $\omega=\mu_{m}\left(\omega^{\prime}\right)$, i.e. 


$$
\begin{aligned}
& \tilde{\omega}=w_{m}\left(\tilde{\omega}^{\prime}\right)+\left(k+h^{v}\right) \omega_{\mu_{m}(0)} \text {. Now } \\
& \begin{aligned}
|\tilde{\omega}|^{2} & =\left|w_{m}\left(\tilde{\omega}^{\prime}\right)+\left(k+h^{v}\right) \omega_{\mu_{m}(0)}\right|^{2} \\
= & \left|\tilde{\omega}^{\prime}\right|^{2}+2\left(k+h^{v}\right)\left[\left\langle w_{m}\left(\tilde{\omega}^{\prime}\right), \omega_{\mu_{m}(0)}\right\rangle+\frac{k+h^{v}}{2}\left|\omega_{\mu_{m}(0)}\right|^{2}\right] \\
= & \left|\tilde{\omega}^{\prime}\right|^{2}+2\left(k+h^{v}\right)\left[\left\langle\tilde{\omega}^{\prime}, \omega_{\mu_{m}(0)}\right\rangle+\frac{k+h^{v}}{2}\left|\omega_{\mu_{m}(0)}\right|^{2}\right] \bmod \left[2\left(k+h^{v}\right)\right] .
\end{aligned}
\end{aligned}
$$

But due to (4.37),

$$
\left\langle\tilde{\omega}^{\prime}, \omega_{\mu_{m}(0)}\right\rangle=-\frac{k}{2}\left|\omega_{\mu_{m}(0)}\right|^{2}+\left\langle\rho, \omega_{\mu_{m}(0)}\right\rangle \bmod [1]
$$

(we use the fact that length squared of elements of the coroot lattice is an even integer).

Equations (10), (11) and (12) imply that

and prove (9).

$$
|\tilde{\omega}|^{2}=\left|\tilde{\omega}^{\prime}\right|^{2} \bmod \left[2\left(k+h^{v}\right)\right]
$$

The second invariance condition (7) was shown in [2] to follow from the matrix relation

$$
S M=M S,
$$

where $S=\left(S_{\omega, \omega^{\prime}}\right), \tilde{\omega}, \tilde{\omega}^{\prime}$ belonging to $\left(k+h^{v}\right) \overline{C_{0}^{\text {aff }}}$,

$$
S_{\omega, \omega^{\prime}}=C \sum_{w \in W} \varepsilon(w) \exp \left[2 \pi i \frac{\left\langle\tilde{\omega}, w\left(\tilde{\omega}^{\prime}\right)\right\rangle}{k+h^{v}}\right],
$$

( $W$ is the Weyl group of $\tilde{G}) \varepsilon(w)=(-1)^{\text {parity(w) }}$ and $C$ being a constant. Note important identities:

$$
\begin{aligned}
S_{\omega, \mu_{m}\left(\omega^{\prime}\right)}= & C \sum_{w \in W} \varepsilon(w) \exp \left[2 \pi i \frac{\left\langle\tilde{\omega} w w_{m}\left(\tilde{\omega}^{\prime}\right)\right\rangle}{k+h^{v}}+2 \pi i\left\langle\tilde{\omega}, w\left(\omega_{\mu_{m}(0)}\right)\right\rangle\right] \\
& =\varepsilon\left(w_{m}\right) \exp \left[2 \pi i\left\langle\tilde{\omega}, \omega_{\mu_{m}(0)}\right\rangle\right] S_{\omega, \omega^{\prime}},
\end{aligned}
$$

and similarly

$$
S_{\mu_{m}(\omega), \omega^{\prime}}=\varepsilon\left(w_{m}\right) \exp \left[2 \pi i\left\langle\tilde{\omega}^{\prime}, \omega_{\mu_{m}(0)}\right\rangle\right] S_{\omega, \omega^{\prime}}
$$

Using these relations and representation (4.44) for the mass matrix, we obtain

$$
\begin{aligned}
(S M)_{\omega, \omega^{\prime}}= & \frac{1}{|Z|} \sum_{m, n} S_{\omega, \mu_{m}\left(\omega^{\prime}\right)} \exp \left[-2 \pi i\left\langle\omega^{\prime}, n \theta\right\rangle+\pi i k\langle m \theta, n \theta\rangle(+\pi i m \wedge n)\right] \\
= & \frac{1}{|Z|} \sum_{m, n} \varepsilon\left(w_{m}\right) \exp \left[-2 \pi i\left(\langle\rho, m \theta\rangle+\langle\omega, m \theta\rangle+\left\langle\omega^{\prime}, n \theta\right\rangle\right)\right. \\
& +\pi i k\langle m \theta, n \theta\rangle(+\pi i m \wedge n)] S_{\omega, \omega^{\prime}},
\end{aligned}
$$

and for the other order (replacing $M$ by its transposition) 


$$
\begin{aligned}
(M S)_{\omega, \omega^{\prime}}= & \frac{1}{|Z|} \sum_{m, n} \exp [-2 \pi i\langle\omega, n \theta\rangle+\pi i k\langle m \theta, n \theta\rangle(+\pi i m \wedge n)] S_{\mu_{m}(\omega), \omega^{\prime}} \\
= & \frac{1}{|Z|} \sum_{m, n} \varepsilon\left(w_{m}\right) \exp \left[-2 \pi i\left(\langle\rho, m \theta\rangle+\left\langle\omega^{\prime}, m \theta\right\rangle+\langle\omega, n \theta\rangle\right)\right. \\
& +\pi i k\langle m \theta, n \theta\rangle(+\pi i m \wedge n)] S_{\omega, \omega^{\prime}} .
\end{aligned}
$$

But, as shown in Appendix 3,

$$
\varepsilon\left(w_{m}\right)=\exp [2 \pi i\langle\rho, m \theta\rangle],
$$

so that equality (14) follows from (18) and (19) by exchange $m \leftrightarrow n$. This completes proof of the modular invariance of partition functions (4.50).

The series of modular invariants that we have obtained here is more general than the one discussed in [2], where the modular invariants were associated with single elements of the center of $\tilde{G}$ (the generators of $Z=Z_{N}$ in our language). Firstly, some central charges $k$ were strangely missed in [2] by requiring that $k|\theta|^{2}$ be an integer, (condition (3.11) of [2]). Secondly, we get pairs of new invariants (one for each periodic vacuum) for the WZW model with group $D_{2 l} / \mathbf{Z}_{2} \times \mathbf{Z}_{2}$. They are associated to a pair of generators of $\mathbf{Z}_{2} \times \mathbf{Z}_{2}$ rather than to a single element of the center.

\section{Examples}

In the present section, we shall give more explicit expressions for the mass matrices of WZW models with non-simply connected groups $\widetilde{G} / Z$. We shall label the weights of group $\tilde{G}$ by $(l+1)$ integers $\left(r_{j}\right), j=0, \ldots, l \equiv \operatorname{rank} \tilde{G}$ such that $\Sigma k_{j}^{v} r_{j}=k$. The correspondence is

$$
\omega=\sum_{j=1}^{l} r_{j} \omega_{j}
$$

where $\omega_{0}=0$ and $\omega_{1}, \ldots, \omega_{l}$ are the fundamental weights. The gain from this somewhat strange labelling is two-fold. First, integrable weights correspond to $\left(r_{j}\right)$ with $r_{j} \geqq 0$. Secondly, due to (4.29),

$$
\begin{aligned}
\mu_{m}(\omega) & \equiv w_{m}(\omega)+k \omega_{\mu_{m}(0)}=\sum_{j=0}^{l} r_{j} w_{m}\left(\omega_{j}\right)+k \omega_{\mu_{m}(0)} \\
& =\sum_{j=0}^{l} r_{j} \omega_{\mu_{m}(j)}-\sum_{j=0}^{l} r_{j} k_{j}^{v} \omega_{\mu_{m}(0)}+k \omega_{\mu_{m}(0)}=\sum_{j=0}^{l} r_{\mu_{m}^{-1}(j)} \omega_{j} .
\end{aligned}
$$

First consider the case $Z=\mathbf{Z}_{N}$. Here $\mu_{m}=\mu^{m}$. Upon replacement in (4.44) of $\theta$ by $-\omega_{\mu(0)}$ (recall that their difference is in the coroot lattice), the mass matrix becomes

$$
M_{\left.\left(r_{j}\right), r_{j}^{\prime}\right)}=\frac{1}{N} \sum_{n, m=0}^{N-1} \exp \left[2 \pi i n \sum_{j=1}^{l} r_{j}^{\prime}\left\langle\omega_{j}, \omega_{\mu(0)}\right\rangle+\pi i k m n\left|\omega_{\mu(0)}\right|^{2}\right] \prod_{j=0}^{l} \delta_{r_{\mu^{m}(j)}, r_{j}^{\prime}}
$$

Notice that in order to compute (3), we only need to know the fundamental weights of $\widetilde{G}$ and the permutation $\mu$ defining a symmetry of the extended Dynkin diagram of $\tilde{G}$ corresponding to the generator of the subgroup $Z$ of the center of $\widetilde{G}$, 


\section{$A_{1}, 1,2, \ldots$}

The integrable weights satisfy $\Sigma r_{j}=k$. Possible subgroups $Z$ of the center $\mathbf{Z}_{l+1}$ are $\mathbf{Z}_{N}$, where $N$ divides $l+1$. The integrability condition for $k$ requires that $k \in 2 \mathbf{Z}$ if $l$ and $(l+1) / N$ are odd and $k \in \mathbf{Z}$ otherwise.

$$
M_{\left(r_{j}\right),\left(r_{j}^{\prime}\right)}=\frac{1}{N} \sum_{m, n=0}^{N-1} \exp \left[-2 \pi i n \sum_{j=1}^{l} j r_{j}^{\prime} / N+\pi i k n m l(l+1) / N^{2}\right] \prod_{j=0}^{l} \delta_{r_{j+m(l+1) / N}, r_{j}^{\prime}},
$$

where $j+m(l+1) / N$ is taken $\bmod [l+1]$.

$B_{1}, 1=2,3, \ldots$

Integrable weights: $r_{0}+r_{1}+2\left(r_{2}+\cdots+r_{l-1}\right)+r_{l}=k . Z=\mathbf{Z}_{2} . k \in \mathbf{Z}$.

$$
M_{\left(r_{j}\right),\left(r_{j}^{\prime}\right)}=\frac{1}{2}\left[\left(1+(-1)^{r_{l}^{\prime}}\right) \delta_{r_{0} r_{0}^{\prime}} \delta_{r_{1} r_{1}^{\prime}}+\left(1+(-1)^{r_{l}^{\prime}+k}\right) \delta_{r_{0} r_{1}^{\prime}} \delta_{r_{1} r_{0}^{\prime}}\right] \prod_{j=2}^{l} \delta_{r_{j}, r_{j}^{\prime}} .
$$

$\mathrm{C}_{1}, \mathrm{l}=3,4, \ldots$

Integrable weights: $\Sigma r_{j}=k . Z=\mathbf{Z}_{2}, k \in 2 \mathbf{Z}$ for $l$ odd and $k \in \mathbf{Z}$ for $l$ even.

$$
M_{\left.\left(r_{j}\right), r_{j}^{\prime}\right)}=\frac{1}{2}\left(1+(-1)^{\sum_{j=1}^{l} j r_{j}^{\prime}}\right) \prod_{j=0}^{l} \delta_{r_{j}, r_{j}^{\prime}}+\frac{1}{2}\left(1+(-1)^{\sum_{j=1}^{l} j r_{j}^{\prime}+k l / 2}\right) \prod_{j=0}^{l} \delta_{r_{l-,}, r_{j}^{\prime}}
$$

$D_{1}, l=4,6,8, \ldots$

Integrable weights: $r_{0}+r_{1}+2\left(r_{2}+\cdots+r_{l-2}\right)+r_{l-1}+r_{l}=k$. Center $=\mathbf{Z}_{2} \times \mathbf{Z}_{2}$. There exist three possible choices of cyclic $Z=Z_{2}$ inside it.

1. $k \in 2 \mathbf{Z}$ if $l$ is not divisible by $4, k \in \mathbf{Z}$ otherwise.

$$
\begin{aligned}
& M_{\left(r_{j}\right),\left(r_{j}^{\prime}\right)}=\frac{1}{2}\left(1+(-1)^{\sum_{j=1}^{1-2} j r_{j}^{\prime}+((l-2) / 2) r_{l-1}^{\prime}+(l / 2) r_{l}^{\prime}}\right) \prod_{j=0}^{l} \delta_{r_{j}, r_{j}^{\prime}} \\
& +\frac{1}{2}\left(1+(-1)^{\sum_{j=1}^{l-2} r r_{j}^{\prime}+((l-2) / 2) r_{l-1}^{\prime}+(l / 2) r_{l}^{\prime}+k l / 4}\right) \prod_{j=0}^{l} \delta_{r_{l-j}, r_{j}^{\prime}} .
\end{aligned}
$$

2. $k \in \mathbf{Z}$.

$$
\begin{aligned}
M_{\left.\left(r_{j}\right), r_{j}^{\prime}\right)}= & \frac{1}{2}\left(1+(-1)^{r_{l-1}^{\prime}+r_{l}^{\prime}}\right) \prod_{j=0}^{l} \delta_{r_{j}, r_{j}^{\prime}} \\
& +\frac{1}{2}\left(1+(-1)^{r_{l-1}^{\prime}+r_{l}^{\prime}+k}\right) \delta_{r_{1} r_{0}^{\prime}} \delta_{r_{0} r_{1}^{\prime}} \prod_{j=2}^{l-2} \delta_{r_{J}, r_{j}^{\prime}} \delta_{r_{l}, r_{l-1}^{\prime}} \delta_{r_{l-1}, r_{l}^{\prime}} .
\end{aligned}
$$

3. $k \in 2 \mathbf{Z}$ if $l$ is not divisible by $4, k \in \mathbf{Z}$ otherwise.

$$
\begin{aligned}
M_{\left(r_{j}\right),\left(r_{j}^{\prime}\right)}= & \frac{1}{2}\left(1+(-1)^{\sum_{j=1}^{l-2} j r_{j}^{\prime}+(l / 2) r_{l-1}^{\prime}+((l-2) / 2) r_{l}^{\prime}}\right) \prod_{j=0}^{l} \delta_{r_{j}, r_{j}^{\prime}} \\
& +\frac{1}{2}\left(1+(-1)^{\sum_{j=1}^{l-2} j r_{j}^{\prime}+(l / 2) r_{l-1}^{\prime}+((l-2) / 2) r_{l}^{\prime}+k l / 4}\right) \delta_{r_{l-1}, r_{0}^{\prime}} \delta_{r_{l}, r_{1}^{\prime}} \\
& \cdot \prod_{j=2}^{l-2} \delta_{r_{l-j}, r_{j}^{\prime}} \delta_{r_{0}, r_{l-1}^{\prime}} \delta_{r_{1}, r_{l}^{\prime} \cdot}
\end{aligned}
$$


$D_{1}, 1=5,7,9, \ldots$

Integrable weights as for $l$ even. Center $=\mathbf{Z}_{4}$.

1. $Z=\mathbf{Z}_{2} . k \in \mathbf{Z}$.

$$
\begin{aligned}
M_{\left(r_{j}\right),\left(r_{j}^{\prime}\right)}= & \frac{1}{2}\left(1+(-1)^{r_{l-1}^{\prime}+r_{l}^{\prime}}\right) \prod_{j=0}^{l} \delta_{r_{j}, r_{j}^{\prime}} \\
& +\frac{1}{2}\left(1+(-1)^{r_{l-1}^{\prime}+r_{l}^{\prime}+k}\right) \delta_{r_{1}, r_{0}^{\prime}} \delta_{r_{0}, r_{1}^{\prime}} \prod_{j=2}^{l-2} \delta_{r_{j}, r_{j}^{\prime}} \delta_{r_{l}, r_{l-1}^{\prime}} \delta_{r_{l-1}, r_{l}^{\prime}} .
\end{aligned}
$$

2. $Z=\mathbf{Z}_{4} . k \in 2 \mathbf{Z}$.

$$
M_{\left(r_{j}\right),\left(r_{j}^{\prime}\right)}=\frac{1}{4} \sum_{m, n=0}^{3} e^{\pi n n\left(\sum_{j=1}^{l-2} j r_{j}^{\prime}+(l / 2) r_{l-1}^{\prime}+((l-2) / 2) r_{l}^{\prime}+(k l / 4) m\right)} \prod_{j=0}^{l} \delta_{r_{\mu^{m}(j)}, r_{j}^{\prime}},
$$

where $\mu$ is the permutation $(l-1, l, l-2, l-3, \ldots, 1,0)$.

$\mathbf{E}_{6}$

Integrable weights: $r_{0}+r_{1}+2 r_{2}+3 r_{3}+2 r_{4}+2 r_{5}+r_{6}=k . Z=\mathbf{Z}_{3}, k \in \mathbf{Z}$.

$$
M_{\left(r_{j}\right),\left(r_{j}^{\prime}\right)}=\frac{1}{3} \sum_{m, n=0}^{2} \exp \left[\frac{2 \pi i}{3} n\left(r_{1}^{\prime}+2 r_{2}^{\prime}+r_{4}^{\prime}+2 r_{6}^{\prime}+2 k m\right)\right] \prod_{j=0}^{6} \delta_{r_{\mu^{m}(j)}, r_{j}^{\prime}}
$$

where $\mu=(1,6,4,3,5,2,0)$.

$\mathbf{E}_{7}$

Integrable weights: $r_{0}+r_{1}+2 r_{2}+3 r_{3}+4 r_{4}+3 r_{5}+2 r_{6}+2 r_{7}=k . Z=\mathbf{Z}_{2}, k \in 2 \mathbf{Z}$.

$$
M_{\left(r_{j}\right),\left(r_{j}^{\prime}\right)}=\frac{1}{2}\left(1+(-1)^{r_{1}^{\prime}+r_{3}^{\prime}+r_{7}^{\prime}}\right) \prod_{j=0}^{7} \delta_{r_{j}, r_{j}^{\prime}}+\frac{1}{2}\left(1+(-1)^{r_{1}^{\prime}+r_{3}^{\prime}+r_{7}^{\prime}+k / 2}\right) \prod_{j=0}^{6} \delta_{r_{\mu(j)}, r_{j}^{\prime}}
$$

where $\mu=(1,0,6,5,4,3,2,7)$.

The last case to consider is

$\mathrm{D}_{1}, \mathrm{I}=\mathbf{4 , 6}, \mathbf{8}, \ldots, Z=\mathrm{Z}_{2} \times \mathrm{Z}_{2} . \mathrm{k} \in \mathbf{2 Z}$.

Replacing in (4.44) $m \theta$ and $n \theta$ by $-\omega_{\mu_{m}(0)}$ and $-\omega_{\mu_{m}(0)}$, we obtain

$$
\begin{aligned}
M_{\left(r_{j}\right),\left(r_{j}^{\prime}\right)}= & \frac{1}{4} \sum_{m, n} \exp \left[2 \pi i \sum_{j=1}^{l} r_{j}^{\prime}\left\langle\omega_{j}, \omega_{\mu_{n}(0)}\right\rangle\right. \\
& \left.+\pi i k\left\langle\omega_{\mu_{m}(0)}, \omega_{\mu_{n}(0)}\right\rangle(+\pi i m \wedge n)\right] \prod_{j=0}^{l} \delta_{r_{\mu_{m}(j)}, r_{j}^{\prime}}
\end{aligned}
$$

More explicitly

$$
\begin{aligned}
M_{\left(r_{j}\right),\left(r_{j}^{\prime}\right)=} & \frac{1}{4}\left(1+(-1)^{r_{l-1}^{\prime}+r_{l}^{\prime}}\right)\left(1+(-1)^{\sum_{j=1}^{1-2} j r_{j}^{\prime}+((l-2) / 2) r_{l-1}^{\prime}+(l / 2) r_{l}^{\prime}}\right) \prod_{j=0}^{l} \delta_{r_{j}, r_{j}^{\prime}} \\
& +\frac{1}{4}\left(1 \pm(-1)^{r_{l-1}^{\prime}+r_{l}^{\prime}+k / 2}\right)\left(1+(-1)^{\sum_{j=1}^{l-2} j r_{j}^{\prime}+((l-2) / 2) r_{l-1}^{\prime}+(l / 2) r_{l}^{\prime}+k l / 4}\right) \prod_{j=0}^{l} \delta_{r_{l-j}, r_{j}^{\prime}}
\end{aligned}
$$




$$
\begin{aligned}
& +\frac{1}{4}\left(1+(-1)^{r_{l-1}^{\prime}+r_{l}^{\prime}}\right)\left(1 \pm(-1)^{\sum_{j=1}^{l-2} j r_{j}^{\prime}+((l-2) / 2) r_{l-1}^{\prime}+(l / 2) r_{l}^{\prime}+k / 2}\right) \delta_{r_{1}, r_{0}^{\prime}} \delta_{r_{0}, r_{1}^{\prime}} \\
& \cdot \prod_{j=2}^{l-2} \delta_{r_{j}, r_{j}^{\prime}} \delta_{r_{l}, r_{l-1}^{\prime}} \delta_{r_{l-1}, r_{l}^{\prime}} \\
& +\frac{1}{4}\left(1 \pm(-1)^{r_{l-1}^{\prime}+r_{l}^{\prime}+k / 2}\right)\left(1 \pm(-1)^{\sum_{j=0}^{l-2} r_{j}^{\prime}+((l-2) / 2) r_{l-1}^{\prime}+(l / 2) r_{l}^{\prime}+k(l-2) / 4}\right) \\
& \cdot \delta_{r_{l-1}, r_{0}^{\prime}} \delta_{r_{l}, r_{1}^{\prime}} \prod_{j=2}^{l-2} \delta_{r_{l-j}, r_{j}^{\prime}} \delta_{r_{0}, r_{l-1}^{\prime}} \delta_{r_{1}, r_{l}^{\prime}}
\end{aligned}
$$

\pm signs correspond to different periodic vacua. Sum over two choices of the sign is a linear combination of previous mass matrices (sum over the three different $Z=\mathbf{Z}_{2}$ choices minus the $Z=1$ solution). Their difference gives a modular invariant linearly independent of the previous ones.

\section{Appendix 1}

Consistent quantization of the WZW models requires certain information about the topology of the group $G$ in which the fields take values. For connected, simply connected, simple, compact groups $G$, the first integral homology groups are

$$
H_{q}(G)=\left\{\begin{array}{lll}
\mathbf{Z} & \text { for } & q=0 \\
0 & \text { for } & q=1 \\
0 & \text { for } & q=2 \\
\mathbf{Z} & \text { for } & q=3
\end{array}\right.
$$

$H_{3}(G)$ is generated by the inclusion $\tilde{g}_{0}$ of $S U(2)\left(\cong S^{3}\right)$ into $G$ on the infinitesimal level described by

$$
\left(\begin{array}{ll}
0 & 1 \\
0 & 0
\end{array}\right) \rightarrow e_{\phi}, \quad\left(\begin{array}{ll}
0 & 0 \\
1 & 0
\end{array}\right) \rightarrow e_{-\phi}, \quad\left(\begin{array}{rr}
1 & 0 \\
0 & -1
\end{array}\right) \rightarrow \phi^{v},
$$

where $\phi$ is the highest root of the Lie algebra $g$ of $G$ [3].

For non-simply connected groups $G=\widetilde{G} / Z$, where $Z$ is a nontrivial subgroup of the center of the covering group $\widetilde{G}$ of the previous type, $H_{0}(G)=\mathbf{Z}$ and $H_{1}(G)=\pi_{1}(G)=Z$. The low homology groups of $G$ are related to the homology of group $Z$ (for the definition of homology of finite groups see [25]). By Corollary XI.7.3. of [18],

$$
H_{q}(G) \cong H_{q}(Z), \quad q=0,1,2,
$$

and there exists an exact sequence

$$
H_{4}(Z) \rightarrow H_{3}(\tilde{G}) \rightarrow H_{3}(G) \rightarrow H_{3}(Z) \rightarrow 0,
$$

where the isomorphism (3) and the last arrow in (4) may be described explicitly [25] (the second arrow in (4) is induced by the canonical projection of $\widetilde{G}$ onto $G$ ). 
For $Z=\mathbf{Z}_{N}, H_{0}(Z)=\mathbf{Z}$ and

$$
H_{q}(Z)= \begin{cases}\mathbf{Z}_{N} & \text { for odd } p \\ 0 & \text { for even } p>0\end{cases}
$$

see [25]. Thus $H_{2}(G)=0$ in this case and there exists an exact sequence

$$
0 \rightarrow \mathbf{Z} \rightarrow H_{3}(G) \rightarrow \mathbf{Z}_{N} \rightarrow 0 .
$$

We shall need a more detailed description of $(6) .1 \in \mathbf{Z}$ (the generator of $H_{3}(\widetilde{G})$ ) is mapped to the homology class $\left[\tilde{g}_{0}\right]$ of the inclusion of $S U(2)$ into $G$ given infinitesimally by (2). An element of $H_{3}(G)$ projecting onto the generator of $\mathbf{Z}_{N}$ may be obtained from the map $\tilde{g}: B \rightarrow G$ defined as follows. Let $B_{1}, B_{2}$ be two distinct copies of $D \times S^{1}(D=$ unit disc $)$. Let $F: S^{1} \times S^{1} \equiv T^{2} \rightarrow T^{2}$,

$$
F\left(e^{i \varphi_{1}}, e^{i \varphi_{2}}\right)=\left(e^{i \varphi_{1}}, e^{i\left(N \varphi_{1}+\varphi_{2}\right)}\right) .
$$

$B$ is the union of $B_{1}$ and $B_{2}$ with the boundaries identified by $F$. It is a closed oriented 3-dimensional manifold. Let $e^{2 \pi i \theta}, \theta \in g$, be the generator of $Z$ and let $g_{1}: D \rightarrow G$ satisfy

$$
\left.g_{1}\right|_{\partial D}=e^{i N \varphi \theta}
$$

(the right-hand side is a contractible loop in $G$ ). Define $\tilde{g} \equiv \tilde{g}_{\theta}$ by putting on $B_{1}$

$$
\tilde{g}_{\theta}\left(r e^{i \varphi_{1}}, e^{i \varphi_{2}}\right)=g_{1}\left(r e^{i \varphi_{1}}\right) e^{i \varphi_{2} \theta},
$$

and on $B_{2}$

$$
\tilde{g}_{\theta}\left(r^{\prime} e^{i \psi_{1}}, e^{i \psi_{2}}\right)=e^{i \psi_{2} \theta} .
$$

A somewhat tedious calculation involving an explicit triangulation of $B$ shows that the homology class $\left[\tilde{g}_{\theta}\right]$ in $H_{3}(G)$ indeed projects via $(6)$ to the generator of $\mathbf{Z}_{N}$. We conclude that for $Z=\mathbf{Z}_{N}$ the homology classes $\left[\tilde{g}_{0}\right]$ and $\left[\tilde{g}_{\theta}\right]$ generate $H_{3}(G)$.

The only case not covered by the above is the possibility $Z=\mathbf{Z}_{2} \times \mathbf{Z}_{2}=$ $\left\{e^{2 \pi i\left(m_{1} \theta_{1}+m_{2} \theta_{2}\right)} \mid m_{i}=0,1\right\}\left(\theta_{1} \neq \theta_{2} \in g\right)$ for $\tilde{G}=D_{2 \mathrm{p}}$. Here $H_{2}(G) \cong H_{2}\left(\mathbf{Z}_{2} \times \mathbf{Z}_{2}\right)=\mathbf{Z}_{2}$ is generated by map $g: T^{2} \rightarrow G$,

$$
g\left(e^{i \varphi_{1}}, e^{i \varphi_{2}}\right)=e^{i\left(\varphi_{1} \theta_{1}+\varphi_{2} \theta_{2}\right)} .
$$

$H_{3}\left(\mathbf{Z}_{2} \times \mathbf{Z}_{2}\right)=\mathbf{Z}_{2} \times \mathbf{Z}_{2} \times \mathbf{Z}_{2}$ and $H_{3}(G)$ enters the exact sequence

$$
\mathbf{Z} \rightarrow H_{3}(G) \rightarrow \mathbf{Z}_{2} \times \mathbf{Z}_{2} \times \mathbf{Z}_{2} \rightarrow 0
$$

with the image of $1 \in \mathbf{Z}$ in $H_{3}(G)$ again equal to the homology class $\left[\tilde{g}_{0}\right]$, and $\left[\tilde{g}_{\theta_{1}}\right],\left[\tilde{g}_{\theta_{2}}\right]$ and $\left[\tilde{g}_{\theta_{1}+\theta_{2}}\right]$ projecting respectively to $(1,0,0),(0,0,1)$ and $(1,1,1)$. Thus $H_{3}\left(D_{2 p} / \mathbf{Z}_{2} \times \mathbf{Z}_{2}\right)$ is generated by $\left[\tilde{g}_{0}\right],\left[\tilde{g}_{\theta_{1}}\right],\left[\tilde{g}_{\theta_{2}}\right]$ and $\left[\tilde{g}_{\theta_{1}+\theta_{2}}\right]$.

Equipped with the knowledge of the generators of $H_{3}(G)$, we turn the consistency requirement (2.2) to the explicit conditions for the coupling constant $k$. For $\tilde{g}=\tilde{g}_{0}$, the integral in (2.2) reduces to the computation of the volume of $S U(2)$ and gives the condition $k \in \mathbf{Z}$ for all groups. For $\tilde{g}=\tilde{g}_{\theta}$, only $\tilde{g}_{\theta} \mid B_{1}$ contributes to the integral. In virtue of the invariance of the 3 -form $\left\langle g^{-1} d g,\left[g^{-1} d g, g^{-1} d g\right]\right\rangle$ 
on $G$ under the right multiplication by $e^{i \varphi_{2} \theta}$,

$$
\begin{gathered}
\int_{D \times S^{1}}\left\langle\tilde{g}^{-1} d \tilde{g},\left[\tilde{g}^{-1} d \tilde{g}, \tilde{g}^{-1} d \tilde{g}\right]\right\rangle=3 i \int_{D}^{2 \pi} \int_{0}^{2 \pi} d \varphi_{2}\left\langle\theta,\left[g_{1}^{-1} d g_{1}, g_{1}^{-1} d g_{1}\right]\right\rangle \\
=-12 \pi i \int_{D} d\left\langle\theta, g_{1}^{-1} d g_{1}\right\rangle=-12 \pi i \int_{\partial D}\left\langle\theta, g_{1}^{-1} d g_{1}\right\rangle=24 \pi^{2} N|\theta|^{2} .
\end{gathered}
$$

Hence $k N / 2|\theta|^{2} \in \mathbf{Z}$. This, together with the integrality, leads to the values of $k$ listed in Sect. 6 for all but one case. The exception is $D_{2 p} / \mathbf{Z}_{2} \times \mathbf{Z}_{2}$, where the condition (2.2) is equivalent to the integrality of $k, k\left|\theta_{1}\right|^{2}, k\left|\theta_{2}\right|^{2}$ and $k\left|\theta_{1}+\theta_{2}\right|^{2}$ and admits $k \in 2 \mathbf{Z}$ for $p$ odd and $k \in \mathbf{Z}$ for $p$ even. In the latter case however, the odd values of $k$ lead to non-unitary theories with complex partition functions.

\section{Appendix 2}

We shall discuss here the construction of the probability amplitudes for the WZW model in a more down-to-earth way than in [9]. Let us focus on the topological part of the amplitude of the field $g$ mapping a compact Riemann surface $\Sigma$ into $G^{\mathrm{C}}$ :

$$
A_{\Sigma}(g)=\exp \left[\frac{i k}{24 \pi} \int_{B}\left\langle\tilde{g}^{-1} d \tilde{g},\left[\tilde{g}^{-1} d \tilde{g}, \tilde{g}^{-1} d \tilde{g}\right]\right\rangle\right],
$$

see (2.1). Equation (1) defines $A_{\Sigma}(g)$ for $g$ homological to zero unambiguously for $k$ satisfying the quantization condition (2.2). $A_{\Sigma}(g)$ is invariant under the orientation preserving reparametrizations of $\Sigma$ and goes into its inverse under the ones changing the orientation. Although $A_{\Sigma}(g)$ is not given as an integral of a local expression over $\Sigma$, its variation is:

$$
\frac{d}{d t} A_{\Sigma}(g)=-\frac{i k}{4 \pi} \int_{\Sigma}\left\langle g^{-1} \frac{\partial g}{\partial t}, d\left(g^{-1} d g\right)\right\rangle .
$$

In the case of $G=D_{2 p} / \mathbf{Z}_{2} \times \mathbf{Z}_{2}$, the only one with non-vanishing second homology group, we should complete the definition of the amplitude by extending $A_{\Sigma}(g)$ to maps $g$ not homologous to zero. Let for $m=\left(m_{1}, m_{2}\right), n=\left(n_{1}, n_{2}\right)$, $g_{m n}: S^{1} \times S^{1} \equiv T^{2} \rightarrow G$ be given by

$$
g_{m n}\left(e^{i \varphi_{1}}, e^{i \varphi_{2}}\right)=e^{i\left(\varphi_{1} m \theta+\varphi_{2} n \theta\right)},
$$

where $m \theta \equiv m_{1} \theta_{1}+m_{2} \theta_{2}$ and $e^{2 \pi i \theta_{1}}, e^{2 \pi i \theta_{2}}$ are the generators of the center of $D_{2 p}$. $g_{(1,0)(0,1)}$ defines the nontrivial element of $H_{2}(G)=\mathbf{Z}_{2}$ (see Appendix 1) and $g_{(2,0)(0,1)}$ is homologous to zero. Proceeding as in (A.1.9) or using directly (2), we obtain

$$
A_{T^{2}}\left(g_{(2,0)(0,1)}\right)=\exp \left[2 \pi i k\left\langle\theta_{1}, \theta_{2}\right\rangle\right]=(-1)^{k}
$$

as $\left\langle\theta_{1}, \theta_{2}\right\rangle=1 / 2 \bmod [1]$. We shall choose $A\left(g_{(1,0)(0,1)}\right)$ as a square root of (4),

$$
A_{T^{2}}\left(g_{(1,0)(0,1)}\right)= \pm e^{\pi i k / 2}
$$

and define for general $g$

$$
A_{\Sigma}(g)= \pm e^{\pi i k[g] / 2} \exp \left[\frac{i k}{24 \pi} \int_{B}\left\langle\tilde{g}^{-1} d \tilde{g},\left[\tilde{g}^{-1} d \tilde{g}, \tilde{g}^{-1} d \tilde{g}\right]\right\rangle\right],
$$


where $[g] \in \mathbf{Z}_{2}$ is the homology class of $g$ and $\partial \tilde{g}=g-[g] g_{(1,0)(0,1)}$ as a 2-chain. It is easy to see that extension (6) preserves the reparametrization invariance of the amplitude and continues to satisfy (2). The freedom of choice of the sign in (5) is in agreement with the results of [9] where different quantizations of the model were labelled by $H^{2}(G, U(1))$. Indeed, the cohomology long exact sequence

$$
\ldots H^{2}(G, \mathbf{R}) \rightarrow H^{2}(G, U(1)) \rightarrow H^{3}(G) \rightarrow H^{3}(G, \mathbf{R}) \ldots
$$

implies that $H^{2}(G, U(1))$ is the torsion part of $H^{3}(G)$ since $H^{2}(G, \mathbf{R})=0$, and the last arrow injects the free part of $H^{3}(G)$. But, by the universal coefficient theorem, the torsion of $H^{3}(G)$ is equal to the torsion of $H_{2}(G)=\mathbf{Z}_{2}$.

To guarantee the reality properties of the theory, see (2.15), we shall additionally require that

$$
A_{\Sigma}\left(g^{*}\right)=\overline{A_{\Sigma}(g)}
$$

Since $g_{(1,0)(0,1)}^{*}$ is an orientation preserving reparametrization of $g_{(1,0)(0,1)}$, this implies the reality of $e^{\pi i k / 2}$ and leaves us with even $k$ 's for which property (6) follows. Noticing that for $m$ proportional to $n, g_{m n}$ is homological to zero modulo $\partial \tilde{g}$, where $\tilde{g}$ does not contribute to (6), and using the reparametrization invariance, we compute

$$
A_{T^{2}}\left(g_{m n}\right)=( \pm)^{m \wedge n}
$$

where $m \wedge n \equiv m_{1} n_{1}-m_{2} n_{1}$.

For $g_{1}=g_{m n}, g_{2}=g_{m^{\prime} n^{\prime}}$, explicit check shows that

$$
\begin{aligned}
A_{T^{2}}\left(g_{1} g_{2}\right)= & ( \pm 1)^{m \wedge n^{\prime}+n \wedge m^{\prime}} \exp \left[\pi i k\left(\left\langle m \theta, n^{\prime} \theta\right\rangle-\left\langle n \theta, m^{\prime} \theta\right\rangle\right)\right. \\
& \left.+\frac{i k}{4 \pi} \int_{T^{2}}\left\langle g_{1}^{-1} d g_{1}, g_{2} d g_{2}^{-1}\right\rangle\right] A_{T^{2}}\left(g_{1}\right) A_{T^{2}}\left(g_{2}\right) .
\end{aligned}
$$

Using Eq. (2), it is easy to verify that (10) holds for any $g_{1}$ and $g_{2}$ in the homotopy class of $g_{m n}$ and $g_{m^{\prime} n^{\prime}}$ respectively.

The holomorphic line bundle $\mathscr{L}$ over $L G^{\mathrm{C}}$ with holomorphic connection is determined by the holonomies of closed paths in $L G^{\mathrm{C}}$ given by the $\mathrm{WZ}$ action (6) on tori. The construction goes as follows.

In each connected component of $L G^{\mathrm{C}}$ we choose a reference point $g *$. Then $\mathscr{L}$ is the set of the equivalence classes of pairs $(\gamma, z)$, where $\gamma$ is a path $\gamma:[0,1] \rightarrow L G^{\mathbf{C}}$ with $\gamma(0)=g *$ and $z \in \mathbf{C}$. Two such pairs $(\gamma, z)$ and $\left(\gamma^{\prime}, z^{\prime}\right)$ are identified provided $\gamma(1)=\gamma^{\prime}(1)$ and

$$
z^{\prime}=A_{T^{2}}\left(\gamma^{\prime-1} * \gamma\right) z
$$

Here $\gamma^{\prime-1}$ denotes the path $\gamma^{\prime}$ run in reverse order and for paths $\gamma_{1}, \gamma_{2}, \gamma_{2}(0)=\gamma_{1}(1)$,

$$
\gamma_{2} * \gamma_{1}(t)=\left\{\begin{array}{lll}
\gamma_{1}(2 t) & \text { for } & t \in\left[0, \frac{1}{2}\right] \\
\gamma_{2}(2 t-1) & \text { for } & t \in\left[\frac{1}{2}, 1\right]
\end{array}\right.
$$

The projection $\pi: \mathscr{L} \rightarrow L G^{\mathbf{C}}$ is given by $\pi[(\gamma, z)]=\gamma(1)$.

Due to the reparametrization invariance of $A_{T^{2}}$, Eq. (11) does not depend on the details of the identification of the closed paths in $L G^{\mathrm{C}}$ with maps from torus 
$T^{2}$ to $G^{\mathrm{C}}$ implied in (11) and defines an equivalence relation. Similarly, different choices of the reference loop $g *$ lead to isomorphic bundles $\mathscr{L}$.

We may now define the parallel transport in $\mathscr{L}$. Given path $\gamma_{1}$ in $L G^{\mathrm{C}}$, the parallel transporter $A\left(\gamma_{1}\right)$ mapping $\mathscr{L}_{\gamma_{1}(0)}$ to $\mathscr{L}_{\gamma_{1}(1)}$ is defined by

$$
A\left(\gamma_{1}\right)[(\gamma, z)]=\left[\left(\gamma_{1} * \gamma, z\right)\right]
$$

(this is readily seen to be well defined using (11)). It satisfies

$$
A\left(\gamma_{1}\right) A\left(\gamma_{2}\right)=A\left(\gamma_{1} * \gamma_{2}\right)
$$

If $\gamma_{1}$ is a closed path then

$$
A\left(\gamma_{1}\right)[(\gamma, z)]=\left[\left(\gamma, A_{T^{2}}\left(\gamma^{-1} * \gamma_{1} * \gamma\right) z\right)\right]=A_{T^{2}}\left(\gamma_{1}\right)[(\gamma, z)],
$$

i.e. $A_{T^{2}}\left(\gamma_{1}\right)$ is the holonomy as desired.

The covariant derivative $D / d t$ along a path $g_{t}$ in $L G^{\mathrm{C}}$ is now easily computed. Let $\psi$ be a section of $\mathscr{L}$. By (11), we may view it as a function $\tilde{\psi}$ on the paths $\gamma$ which satisfies

$$
\tilde{\psi}\left(\gamma^{\prime}\right)=A_{T^{2}}\left(\gamma^{\prime-1} * \gamma\right) \tilde{\psi}(\gamma)
$$

(smooth $\tilde{\psi}$ satisfying (16) defines a section $\psi, \psi(\gamma(1))=[(\gamma, \widetilde{\psi}(\gamma))])$. Denote by $\gamma_{t t^{\prime}}$ the path $g_{t}$ run from $t^{\prime}$ to $t$. Thus

$$
\begin{aligned}
\frac{D}{d t} \psi\left(g_{t}\right) & =\left.\frac{d}{d t^{\prime}}\right|_{t^{\prime}=t} A\left(\gamma_{t t^{\prime}}\right) \psi\left(g_{t^{\prime}}\right) \\
& =\left.\frac{d}{d t^{\prime}}\right|_{t^{\prime}=t}\left[\left(\gamma_{t t^{\prime}} * \gamma_{t^{\prime}}, \tilde{\psi}\left(\gamma_{t^{\prime}}\right)\right)\right] \\
& =\left.\frac{d}{d t^{\prime}}\right|_{t^{\prime}=t}\left[\left(\gamma_{t}, A_{T^{2}}\left(\gamma_{t}^{-1} * \gamma_{t t^{\prime}} * \gamma_{t^{\prime}}\right) \tilde{\psi}\left(\gamma_{t^{\prime}}\right)\right)\right] \\
& \equiv\left(\gamma_{t}, \frac{D}{d t} \widetilde{\psi}\left(\gamma_{t}\right)\right),
\end{aligned}
$$

where $\gamma_{t}:[0,1] \rightarrow L G^{\mathrm{C}}, \gamma_{t}(0)=g *, \gamma_{t}(1)=g_{t}$. The derivative of $A_{T}$ is computed using Eq. (2). Noting that the contribution from $\gamma_{t t^{\prime}}$ vanishes as $t^{\prime} \rightarrow t$, we obtain

$$
\frac{D}{d t} \tilde{\psi}\left(\gamma_{t}\right)=\left(\frac{d}{d t}-\frac{i k}{4 \pi} \int_{[0,1] \times S^{1}}\left\langle\gamma_{t}^{-1} \frac{\partial \gamma_{t}}{\partial t}, d\left(\gamma_{t}^{-1} d \gamma_{t}\right)\right\rangle\right) \tilde{\psi}\left(\gamma_{t}\right) .
$$

The action of the WZW model includes, apart from the topological term, also the standard sigma-model part. Identifying a path $g:\left[\tau_{1}, \tau_{2}\right] \rightarrow L G^{\mathrm{C}}$ between euclidean times $\tau_{1}$ and $\tau_{2}$ with a field configuration on the annulus $\Sigma=\left\{z \mid e^{\tau_{1}} \leqq\right.$ $\left.z \leqq e^{\tau_{2}}\right\}$ by the exponential map, we put

$$
e^{-S_{\Sigma}(g)}=\exp \left[\frac{i k}{4 \pi} \int_{\Sigma}\left\langle g^{-1} \partial g, g^{-1} \bar{\partial} g\right\rangle\right] A(g) \equiv e^{-S_{\Sigma}^{0}(g)} A(g) .
$$

$\exp \left[-S_{\Sigma}(g)\right]$ is an element of $\mathscr{L}_{g\left(\tau_{2}\right)} \otimes \mathscr{L}_{g\left(\tau_{1}\right)}^{*}$.

It is convenient to use $\exp \left[-S_{\Sigma}(g)\right]$ rather than $[(\gamma, z)]$ to represent the 
elements of $\mathscr{L}$. A general element of $\mathscr{L}_{g\left(\tau_{2}\right)}$ is given by

$$
e^{-S_{\Sigma}(g)} \mu, \mu \in \mathscr{L}_{g_{*}} \text {, }
$$

for $g\left(\tau_{1}\right)$ fixed at $g *$. If $\mu=\left[\left(\gamma_{0}, z_{0}\right)\right]$ then

$$
e^{-S_{\Sigma}(g)} \mu=\left[\left(g * \gamma_{0}, e^{-S_{\Sigma}^{0}(g)} z_{0}\right)\right]
$$

in the previous representation.

Equation (18) for the covariant derivative gives immediately for a homotopy $g_{t}$,

$$
\begin{aligned}
\frac{D}{d t} e^{-S_{\Sigma}\left(g_{t}\right)} & =\left[\frac{i k}{4 \pi} \int_{\Sigma} \frac{d}{d t}\left\langle g_{t}^{-1} \partial g_{t}, g_{t}^{-1} \bar{\partial} g_{t}\right\rangle-\frac{i k}{4 \pi} \int_{\Sigma} \frac{d}{d t}\left\langle g_{t}^{-1} \frac{\partial g_{t}}{\partial t}, d\left(g_{t}^{-1} d g_{t}\right)\right\rangle\right] e^{-S_{\Sigma}\left(g_{t}\right)} \\
& =\left[\frac{i k}{2 \pi} \int_{\Sigma}\left\langle\partial\left(g_{t}^{-1} \frac{\partial g_{t}}{\partial t}\right), g_{t}^{-1} \bar{\partial} g_{t}\right\rangle-\frac{i k}{4 \pi} \int_{\partial \Sigma}\left\langle g_{t}^{-1} \frac{\partial g_{t}}{\partial t}, g_{t}^{-1} d g_{t}\right\rangle\right] e^{-S_{\Sigma}\left(g_{t}\right)} .
\end{aligned}
$$

On the identity component of $L G^{\mathrm{C}}$, we may take $g *$ a constant and represent the bundle $\mathscr{L}$ with the help of action $S_{D}$ on the disc $D=\{z|| z \mid \leqq 1\}$ by putting $\tau_{1}=-\infty$ and $\tau_{2}=0$. Bundle $\mathscr{L}$ restricted to constant loops is canonically trivialized by the mapping $[(\gamma, z)] \rightarrow\left(\gamma(1), A_{P \mathbf{C}^{1}}(\gamma) z\right) \in G^{\mathbf{C}} \times \mathbf{C},(\gamma(0)$ and $\gamma(1)$ being constant loops, $\gamma$ defines a map from $\mathbf{C} P^{1} \rightarrow G^{\mathbf{C}}$ ). Hence we may take $\exp \left[-S_{D}(g)\right] \in \mathscr{L}_{g \mid \partial D}$ canonically and let the value of $g$ at $z=0$ be arbitrary. It is easy to see that formula (22) still holds for $\Sigma$ replaced by $D$.

On the nontrivial sectors, it is convenient to use the annulus $A=\{z|1 \leqq| z \mid \leqq 2\}$ and fix $\left.g\right|_{|z|=2}=g * \mathscr{L}_{g|| z \mid=1}$ is hence represented by elements $\mu \exp \left[S_{A}(g)\right]$ with $\mu \in \mathscr{L}_{g}$. To obtain the PW formulae (2.10) and (2.11), write

or

$$
e^{-S_{A}(g h)} e^{S_{A}(g)}=e^{-S_{A}^{0}(h g)+S_{A}^{0}(g)} A_{T^{2}}\left(h g * g^{-1}\right)
$$

and use (10).

$$
e^{S_{A}(g)-S_{A}(g h)}=e^{S_{A}^{0}(g)-S_{A}^{0}(g h)} A_{T^{2}}\left(g^{-1} * g h\right),
$$

The classical symmetries (1.1) of the WZW theory act on the (complexified) configuration space $L G^{\mathrm{C}}$ of the model by left and right multiplication by the elements of $L \widetilde{G}^{\mathrm{C}}$. The quantum action of the symmetries comes from the appropriate lift (geometric quantization!) of this action to bundle $\mathscr{L}$. Its infinitesimal generators $J_{n}(x)$ and $\bar{J}_{n}(x), x \in g$, (see Sect. 3 ), act on analytic sections $\psi$ of $\mathscr{L}$ by

and

$$
\begin{aligned}
\left(J_{n}(x) \psi\right)(g)= & \left.\frac{1}{i} \frac{D}{d t}\right|_{t=0} \psi\left(e^{-t t e^{i n \varphi} x} g\right) \\
& +\left[\frac{k i}{4 \pi} \int_{0}^{2 \pi}\left\langle x e^{i n \varphi}, \frac{\partial g}{\partial \varphi} g^{-1}\right\rangle d \varphi\right] \psi(g),
\end{aligned}
$$

$$
\begin{aligned}
\left(\bar{J}_{n}(x) \psi\right)(g)= & \left.\frac{1}{i} \frac{D}{d t}\right|_{t=0} \psi\left(g e^{i t e^{-i n \varphi} x}\right) \\
& +\left[\frac{k i}{4 \pi} \int_{0}^{2 \pi}\left\langle x e^{-i n \varphi}, g^{-1} \frac{\partial g}{\partial \varphi}\right\rangle d \varphi\right] \psi(g) .
\end{aligned}
$$


Using the definition of the covariant derivative given here, one can check that $J_{n}$ 's and $\bar{J}_{n}$ 's satisfy the commutation relations (3.7) of the Kac-Moody algebra and give the infinitesimal versions of the global actions of the Kac-Moody groups defined in Sect. 3 for the simply connected groups and in Sect. 4 for the non-simply connected ones.

\section{Appendix 3}

We include here the standard proof of existence of the affine Weyl group element needed in Sect. 4 and of some identities which were used in the proof of modular invariance. The affine Weyl group elements $\tilde{w}$ represented by $e^{i \varphi \sigma} w \in L G$, where $\sigma \in Q\left(R^{v}\right)$ and $w \in G$ defines a finite Weyl group element, act on affine roots $\tilde{\alpha}=\alpha+n$ according to $e_{\tilde{w}(\tilde{x})}=\tilde{w} e_{\tilde{\alpha}} \tilde{w}^{-1}$ readily computed to give

$$
\tilde{w}(\tilde{\alpha})=w(\alpha)+n+\langle w(\alpha), \sigma\rangle .
$$

For $\theta \in Q^{*}(R)$, we want to find the unique affine Weyl group element $\tilde{w}$ such that

$$
\tilde{w}^{-1}(\tilde{\alpha}-\langle\alpha, \theta\rangle)=w^{-1}(\alpha)+n-\langle\alpha, \theta+\sigma\rangle>0
$$

for each positive root $\tilde{\alpha}=\alpha+n$, see (4.23). Let $A$ be the (finite) set of the negative affine roots $\tilde{\beta}=\tilde{\alpha}-\langle\alpha, \theta\rangle \equiv \varphi(\tilde{\alpha})$ with $\tilde{\alpha}\rangle 0$. The set $-A$ contains at least one simple affine root $\tilde{\alpha}_{i}$ if it is non-empty. Indeed, if for all simple $\widetilde{\beta}_{j}, \varphi^{-1}\left(-\widetilde{\beta}_{j}\right)<0$, then $\varphi^{-1}(\tilde{\beta})<0$ for all $\tilde{\beta}<0$ and $A$ is empty. Since the reflections $r_{\tilde{\alpha}_{i}}$ in simple affine roots change the sign of $\tilde{\alpha}_{i}$ and map other positive affine roots into positive ones,

$$
\left|r_{\tilde{x}_{i}} A\right|=|A|-1,
$$

where $|\cdot|$ denotes the cardinality. By iteration, we obtain the element $\tilde{w}^{-1}$ with the required properties. Its uniqueness follows from the fact that the only affine Weyl group element mapping positive roots into the positive ones is the identity.

As explained in Sect. 4 the class of $\theta$ in $Q^{*}(R) / Q\left(R^{v}\right)$ uniquely determines an automorphism $\mu$ of the extended Dynkin diagram such that the action of $w\left(\tilde{w}=e^{i \varphi \sigma} w\right)$ on the fundamental weights $\omega_{0}=0, \omega_{1}, \ldots, \omega_{l}$ is

$$
w\left(\omega_{j}\right)+k_{j}^{v} \omega_{\mu(0)}=\omega_{\mu(j)},
$$

see (4.29). Let $\rho$ denote half the sum of the positive roots of the finite dimensional Lie algebra. It is well known that $\rho=\omega_{1}+\cdots+\omega_{l}$ and (4) implies that

compare (5.8).

$$
\rho-w(\rho)=h^{v} \omega_{\mu(0)},
$$

We shall prove now the following formulae for the length $l(w)$ of the finite Weyl group element $w$ :

$$
l(w)=2\left\langle\rho, \omega_{\mu(0)}\right\rangle=h^{v}\left|\omega_{\mu(0)}\right|^{2} .
$$

This will imply (5.10) and (5.20) $\left(\varepsilon(w)=e^{\pi i l(w)}\right)$. Replacing $\theta$ by $-\omega_{\mu(0)}$ above and repeating the preceding construction, we obtain $\tilde{w}=w$ and $l(w)=|A|$, where the set $A$ contains only the roots of the finite Lie algebra. For $\alpha<0,\left\langle\alpha, \omega_{\mu(0)}\right\rangle \leqq 0$ 
and $\left\langle\alpha, \omega_{\mu(0)}\right\rangle=0$ or -1 only,

Thus

$$
A=\left\{\alpha<0 \mid\left\langle\alpha, \omega_{\mu(0)}\right\rangle=-1\right\} .
$$

$$
l(w)=-\sum_{\alpha \in A}\left\langle\alpha, \omega_{\mu(0)}\right\rangle=\sum_{\alpha>0}\left\langle\alpha, \omega_{\mu(0)}\right\rangle=2\left\langle\rho, \omega_{\mu(0)}\right\rangle .
$$

On the other hand,

and

$$
\sum_{\alpha>0}(-\alpha)=\sum_{\alpha \in A} \alpha-\sum_{\substack{\alpha>0 \\\left\langle\alpha, \omega_{\mu(0)}\right\rangle=0}} \alpha
$$

$$
\sum_{\alpha>0} w(\alpha)=\sum_{\alpha \in A} \alpha+\sum_{\substack{\alpha>0 \\\left\langle\alpha, \omega_{\mu(0)}\right\rangle=0}} \alpha
$$

(use inverted (2)).

Thus

$$
\sum_{\alpha \in A} \alpha=w(\rho)-\rho=-h^{v} \omega_{\mu(0)},
$$

where we have used Eq. (5). Taking the scalar product with $\omega_{\mu(0)}$ gives the other equality in (6).

Acknowledgements. We would like to thank D. Bernard for discussions and C.R.M. at Universite de Montréal for hospitality. K. Gawẹdzki and A. Kupiainen also thank the I.T.P. at U.C. Santa Barbara where part of this work was carried out under the support of NSF grant No. PHY82-17853, supplemented by funds from NASA.

\section{References}

1. Abouelsaood, A., Gepner, D.: Phys. Lett. B 176, 380-386 (1986)

2. Bernard, D.: Nucl. Phys. B288, 628-648 (1987)

3. Bott, R.: Bull. Soc. Math. France 84, 251-281 (1956)

4. Bouwknegt, P., Nahm, W.: Phys. Lett. B184, 359-362 (1987)

5. Callan, C. G., Dashen, R. F., Gross, D. J.: Phys. Lett. 63B, 334 (1976)

6. Cappelli, A., Itzykson, C., Zuber, J.-B.: The A-D-E classification of minimal and $A_{1}^{(1)}$ conformal invariant theories. Commun. Math. Phys. 113, 1-26 (1987)

7. Felder, G., Gawędzki, K., Kupiainen, A. The spectrum of Wess-Zumino-Witten models, to appear in Nucl. Phys. B

8. Fröhlich, J.: Lectures at Cargèse Summer School, 1987

9. Gawędzki, K.: Topological actions in two-dimensional quantum field theories, IHES preprint, 1987

10. Gepner, D.: Nucl. Phys. B287, 111-130 (1987)

11. Gepner, D., Witten, E.: Nucl. Phys. B278, 493-549 (1986)

12. Jackiw, R., Rebbi, C.: Phys. Rev. Lett. 37, 172 (1976)

13. Kac, V. G.: Infinite dimensional Lie algebras. Cambridge: Cambridge University Press 1985

14. Kac, V. G., Peterson, D. H.: In Arithmetics and geometry, Vol. 2, pp.141-166. Boston: Birkhäuser 1983

15. Knizhnik, V., Zamolodchikov, A. B.: Nucl. Phys. B247, 83-103 (1984)

16. Kodaira, K.: Complex manifolds and deformation of complex structures, Berlin, Heidelberg, New York: Springer 1986

17. Kostant, B.: In: Lectures Notes in Mathematics, Vol. 170, pp. 87-208. Berlin, Heidelberg, New York: Springer 1970 
18. Mac Lane, S.: Homology. Berlin, Heidelberg, New York: Springer 1963

19. Maurin, K.: General eigenfunctions expansions and unitary representations of topological groups, PWN, Warsaw, 1968

20. Mickelsson, J.: Commun. Math. Phys. 110, 173-184 (1987)

21. Olive, D. I., Goddard, P.: Lectures at Srni Winter School, 1985

22. Polyakov, A. M., Wiegmann, P. B.: Phys. Lett. B141, 223-228 (1984)

23. Pressley, A., Segal, G.: Loop groups. Oxford: Clarendon Press 1986

24. Ramadas, T. R.: Commun. Math. Phys. 93, 355-365 (1984)

25. Séminaire, H...Cartan, Exposés $1^{\circ}-4^{\circ}$, ENS, 1950/1951

26. Simms, D. J., Woodhouse, N. M. J.: Lecture Notes in Physics, Vol. 53, Berlin, Heidelberg, New York: Springer 1976

27. Witten, E.: Commun. Math. Phys. 92, 455-472 (1984)

28. Wudka, J.: Nucl. Phys. B288, 649-658 (1987)

29. Zamolodchikov, A. B., Fateev, V. A.: Sov. Phys. JETP 62, 215-225 (1985)

Communicated by A. Jaffe

Received December 18, 1987 